\title{
Les démons comme agents de destruction, entre animaux et maladies
}

\author{
1 \\ Resheph, Deber, Qeteb et autres agents de malheurs dans la \\ Bible hébraïque
}

Un premier scénario clairement identifiable comme démoniaque dans la Bible hébraïque est composé de plusieurs textes qui présentent une série de puissances responsables d'épidémies et de fléaux. Ces puissances interviennent dans des contextes différents et ne font pas l'objet d'une description détaillée. Toutefois, elles partagent un certain nombre de caractères récurrents qui justifient une mise en réseau des textes concernés ainsi qu'une analyse de leurs échos mutuels. Un premier passage provient du Pentateuque : il s'agit notamment du chapitre 32 de Deutéronome, versets 22-24. Ici, Moïse, à l'entrée du pays de Canaan, annonce aux Israélites les châtiments qui feront nécessairement suite à leur infidélité : Yhwh, en colère, accumulera des malheurs contre eux et leur lancera «ses flèches » parmi lesquelles figurent Resheph, littéralement « la flamme », qui les dévorera, et Qețeb, « le fléau », qui est défini comme « amer » ou « venimeux » $($ marîrî $)$ :

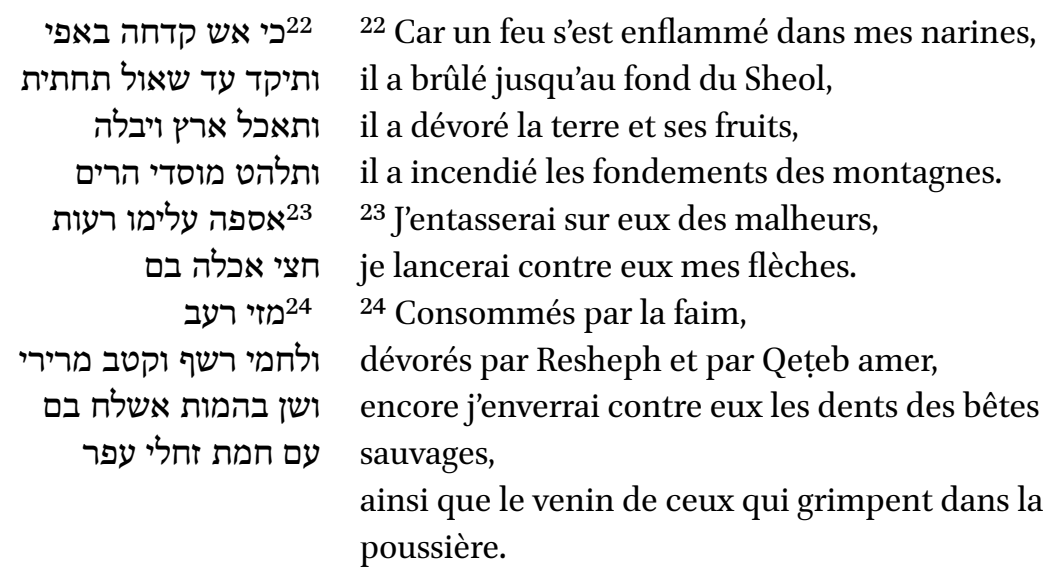

Un deuxième passage se trouve dans la théophanie d'Hab 3 (appelé Psaume d'Habacuc), qui aux versets $3-5$ décrit la marche de Yhwh montant vers 
Jérusalem depuis les plaines de Teman et les monts de Paran, accompagné par ses acolytes Deber et Resheph :

\begin{tabular}{|c|c|}
\hline 3 ${ }^{3}$ & ${ }^{3} \mathrm{I}$ \\
\hline וקדוש מה רפארן & n (Pause). \\
\hline כסה שמים הודו & Sa majesté couvre le ciel, \\
\hline ותהלתו מלאה הארץ & plit la terre. \\
\hline 4 ונגה כאור תהיה & ${ }^{4}$ Sor \\
\hline 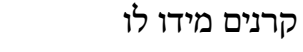 & e sa mair \\
\hline 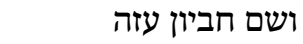 & et là est le secret de sa force. \\
\hline 5 & $\begin{array}{l}{ }^{5} \text { Devant lui marche Deber, } \\
\text { et Resheph sort sur ses pas. }\end{array}$ \\
\hline
\end{tabular}

Deber et Qețeb sont encore mentionnés dans le Ps 91,3-6 (= 9o, 3-6 LXX) où l'orant invoque l'aide et la protection de la divinité contre « la peste (Deber) qui marche dans l'ombre » et contre «le fléau (Qețeb) qui ravage pendant le jour ». Ici Deber et Qețeb sont présentés comme deux puissances malfaisantes contre lesquelles on invoque l'assistance de Yhwh :

\begin{tabular}{|c|c|}
\hline מפח יקוש יצילך $3{ }^{3}$ & $\begin{array}{l}{ }^{3} \text { C'est lui (scil. Yhwh) qui te délivre } \\
\text { du filet du chasseur }\end{array}$ \\
\hline מדבר הוות & et de la peste destructrice. \\
\hline 4 4 באברתו יסך לך & ${ }^{4}$ De ses ailes il te protège, \\
\hline ותחת כנפיו תחסה & et sous ses plumes tu te réfugies. \\
\hline 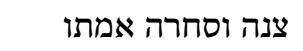 & Sa fidélité est un bouclier et une armure. \\
\hline 5 לא תירא מפחד ק & ${ }^{5} \mathrm{Tu}$ ne craindras ni la terreur de la nuit, \\
\hline 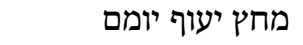 & ni la flèche qui vole au grand jour, \\
\hline 6 - 1דבר באפל יהלך & ${ }^{6}$ ni Deber qui rôde dans l'ombre, \\
\hline מקטב ישוד צהרים & ni Qețeb qui ravage en plein midi. \\
\hline
\end{tabular}

On ajoutera également à cette analyse Ex 12 (versets 13 et 23), où le mašhît, littéralement «l'exterminateur », massacre sur injonction divine les premiers-nés

1 Je comprends ici tahillâ dans le sens d'« éclat», «rayonnement», plutôt que dans le sens traditionnel de «louange », sur la base de la racine $h l l$, «briller» et des parallèles entre 3 b et 4. Sur l'éclat qui entoure la manifestation du dieu au Proche Orient ancien, voir le travail classique de Cassin 1968 et, plus récemment, Aster 2012. Les remarques de Francis Andersen (2001, p. 294) d'après lequel l'apparition de la divinité suscite la louange du peuple dans plusieurs traditions babyloniennes et égyptiennes, ne me paraît pas tenir suffisamment compte des expressions parallèles dans les versets 3 et 4 , qui font clairement référence à des attributs de la divinité. 
des Égyptiens alors qu'il épargne les maisons des Israélites, lesquels, en suivant les instructions données par Yhwh, avaient aspergé leurs portes avec le sang d'une bête égorgée. Des «anges de malheur » sont enfin mentionnés dans le Ps 78 (= 77 LXX), l'un des psaumes désignés comme « historiques » qui résume les traditions de l'Exode.

Ces puissances partagent entre elles un certain nombre de traits. Tout d'abord, il faut remarquer qu'elles sont douées de noms «parlants». Ainsi Qețeb signifie «plaie», Deber «peste», Resheph « flamme», mašhît «le destructeur », etc. Bien que leur étymologie ne soit pas toujours certaine ou assurée, il semble cependant évident que leurs noms sont transparents et qu'ils font référence soit à la fonction de ces puissances, soit à l'épidémie dont elles sont considérées responsables ${ }^{2}$. Le fait que ces noms puissent être également employés comme noms communs a parfois été utilisé comme argument pour nier leur nature divine ou démoniaque : il s'agirait seulement de personnifications occasionnelles des phénomènes naturels ou des maladies ${ }^{3}$. Le problème a été particulièrement discuté pour certaines occurrences de Resheph ${ }^{4}$, notamment lorsque le mot apparaît au pluriel. En effet, dans quelques cas, il apparaît difficile de savoir s'il s'agit d'un agent propre et véritable ou tout simplement de flammes ou de flèches.

À cet égard, une certaine prudence est de mise. Il faut effectivement s'abstenir de reconnaitre un démon de la peste derrière toutes les occurrences du mot deber. Un certain nombre d'usages « figés » sont attestés dans la Bible hébraïque, notamment dans le livre de Jérémie qui utilise souvent la formule stéréotypée selon laquelle le dieu punit, visite ou fait mourir «par épée, famine, peste $»^{5}$. Il faut, en outre, remarquer que dans ces passages deber est presque toujours précédé de l'article déterminatif. La présence de l'article pourrait indiquer que le mot doit être compris dans son sens générique et littéral. Un autre passage à écarter est Is 28,2 : ici qetẹb figure en tant qu'apposition de śa'ar, « tempête », dans une similitude qui évoque plusieurs phénomènes météorologiques, tels que la grêle et l'orage. Au lieu de voir ici une puissance démoniaque, il faudrait plutôt, en accord avec la majorité des chercheurs, donner à qețeb une valeur générique de «destruction ${ }^{6}$. En ce qui concerne Resheph, une signification

2 À propos de la différence entre «transparence » sémantique et méthode étymologique les remarques de James Barr (1968, p. 17) sont encore très utiles.

3 Notamment par Blair 2009, p. 109, mais voir encore, récemment, Kató 2012.

4 Ainsi Münnich 2013, p. 224-237.

5 Jer 14,$12 ; 21,6-7$ et $9 ; 27,8$ et $13 ; 28,8 ; 29,17-18 ; 32,34$ et $36 ; 34,17 ; 38,2 ; 42,17-22 ; 44,13$. Voir également la variante « guerre, famine, peste » en Jer 35,8 .

6 «Ruineux» : Wildberger 1982, p. 1041-1043; Watts, 1985-1987, p. 358-36o ; Beuken 2010, p. $5^{8}$; Roberts 2015, p. 342. 
de rəšāpîm comme «flammes » ou «flèches ardentes» (rišpê 'ěš) se retrouve également à l'intérieur d'une similitude poétique entre l'amour et le Shéol, en Cant 8,6. Dans ce passage, malgré l'évocation d'un imaginaire mythique par la référence au Shéol et aux « grandes eaux » (verset 7), il apparaît difficile de voir une véritable puissance divine à l'œuvre dans les «flammes », qui correspondent plutôt à une métaphore assez usuelle pour décrire la passion amoureuse ${ }^{7}$. Toutefois, l'écart entre un usage au sens «propre » et « commun » de ces noms ne doit pas être surestimé. Dans les cas qu'on examinera par la suite, le contexte indique clairement qu'il s'agit d'agents divins en action qui peuvent se manifester occasionnellement à travers des catastrophes naturelles. Par ailleurs, les parallèles proche-orientaux confirment que certaines puissances démoniaques peuvent avoir des noms transparents indiquant des fléaux ou des nomina agentis construits sur des actions associées de diverses manières à la destruction ${ }^{8}$. Un bon exemple de la manière ancienne de concevoir la relation étroite et, de fait, métonymique entre le fléau et l'agent qui en est responsable se trouve dans le chapitre 24 du deuxième livre de Samuel. Ici Yhwh envoie la peste (deber) en Israël à cause de David qui avait demandé un recensement du peuple $(2 S 24,15)$. La manière dont le fléau s'abat sur le peuple est décrite aux versets suivants (versets 16-17) par l'action du «messager qui extermine le peuple» (hammal'äk hammašhît bāâmm).

Deuxièmement, ces puissances sont souvent mises en rapport avec la cour céleste. Elles apparaissent dans des théophanies divines, dont la mieux connue est, probablement, celle d'Hab 3,1-59 , et peuvent être explicitement qualifiées de «messagères» de la divinité ${ }^{10}$. Certaines sont même explicitement liées au cortège militaire de Yhwh qui a parfois été mis en parallèle avec les armées des dieux mésopotamiens de la peste, Nergal et Erra ${ }^{11}$. Ces passages peuvent aussi présenter des liens intertextuels évidents : un cas particulièrement intéressant est la réécriture de l'épisode de la peste en $2 \mathrm{~S} 24$ dans le premier livre des Chroniques (1 Ch 21). Un autre exemple est le Ps 78 (= $77 \mathrm{LXx})$ qui résume l'histoire d'Israël. Les versets $44^{-}{ }^{1}$ mentionnent les plaies d'Égypte dans un ordre différent de celui de l'Exode mais néanmoins en dialogue avec le récit

$7 \quad$ Pace Pope, 1977 , p. 670. Pour des arguments supplémentaires contre l'interprétation démonique de Resheph, voir Wilson-Wright 2015, p. 340.

8 Voir supra, p. 29, 38-42, ainsi que les exemples grecs, p. 53-55.

9 Hab $3,1-5$.

$10 \quad \operatorname{Ex}_{12} ; 2 \mathrm{~S} 24,6 ; 1 \mathrm{Ch} 21,15 ;$ Ps $78,48-51$.

11 Hab 3,1-5, à comparer avec Deut 33,1-3, et Deut 32,22-24. Sur l'imaginaire partagé par Hab 3,4 et Deut 33,2, voir, récemment, Wearne 2014. Pour la comparaison du cortège de Yhwh avec celui de Nergal et Erra, on peut se référer, entre autres, à Andersen 2001, p. 300-307. 
d'Ex 7-12. Les versets 49-51 closent la séquence des plaies en citant la peste et la mort des premiers-nés :

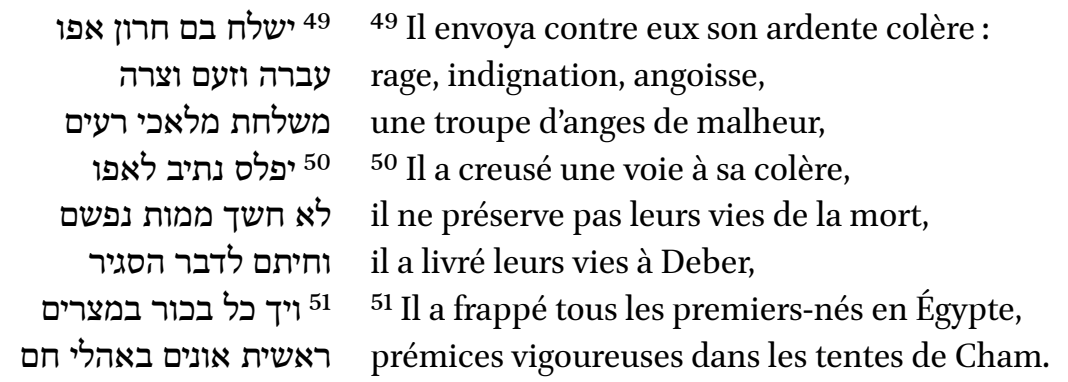

La mention des «messagers de malheurs » au verset 49 est à mettre en relation avec la mort des premiers-nés au verset $5^{1}$ («il a frappé tous les premiers-nés en Égypte»). Ce passage paraît, en effet, évoquer l'envoi du destructeur qui frappe les maisons des Égyptiens en Ex 12,23 ${ }^{12}$.

Troisièmement, il faut observer que dans tous les cas examinés ces puissances sont la manifestation de la colère de Yhwh. En Deut 32,23-24, le feu de la colère de Yhwh «enflamme ses narines » et le pousse à envoyer contre les Israélites infidèles ses agents de destruction, Resheph et Qețeb. Dans le Ps 78, Yhwh « creuse un passage à sa colère » ${ }^{13}$ par le biais de messagers de malheurs. Cette colère, d'ailleurs, peut être elle-même conçue comme une émanation de la divinité qui, parfois, échappe à son contrôle: en Num 17, par exemple, la fureur divine d'abord déchaînée contre les fils d'Aaron aurait risqué d'exterminer tous les Israélites si elle n'avait pas été arrêtée par un rituel apotropaïque accompli par Aaron ${ }^{14}$. En 2 S 24,1, la colère de Yhwh « s'enflamme » contre Israël et incite David à agir contre l'intérêt de son peuple. Le fait qu'elle soit remplacée par Satan dans la version du récit transmise par les Chroniques ${ }^{15}$ démontre une équivalence fonctionnelle entre ces deux agents de la cour céleste.

Enfin, il n'est pas rare que ces puissances interviennent ensemble, en couple ou par groupes, en tant que membres de l'armée de Yhwh. Deut 32,24 mentionne Resheph et Qețeb, tandis que la théophanie d'Habacuc présente Resheph et Deber marchant à côté de Yhwh. Un autre texte clé pour cette analyse est le Ps 91 (= 9o LXX) où Deber et Qețeb figurent parmi les périls qui

12 Voir, déjà, Delitzsch 1888, 426. Sur la nature secondaire de ces versets $49 \mathrm{~b}-5$ oa, voir Spieckermann 1989, p. 137 ; Hossfeld et Zenger 2011, p. 288.

13 Ps 78,5 .

14 Voir à ce sujet Nihan 2015, p. 100-102.

151 Ch 21,1, voir § 8.1.1. 
terrorisent l'orant, lequel cherche son refuge en Yhwh. Comme nous le verrons par la suite, Resheph n'est pas nommé dans ce cas, néanmoins sa présence ne peut pas être complètement exclue. Le Ps 78 , pour sa part, mentionne au verset 49a «furie, malédiction et angoisse » qui sont définies comme une «troupe d'anges de malheurs», et ici le terme rare mišlaḥat a une connotation militaire explicite ${ }^{16}$. L'arrivée de ces messagers est anticipée par l'annonce d'une punition qui s'abattra sur le bétail, abandonné aux flammes ou aux « foudres » (rəšāâim, verset 48), et sur les humains, livrés à la peste (deber, verset 50). Ces passages pourraient être interprétés de manière simplement naturaliste car ils rappellent deux plaies mentionnées dans l'Exode : respectivement la grêle qui tombe sur les Égyptiens mêlée au feu ('ěš, en Ex 9,24) et la peste qui frappe leur bétail (deber, en Ex 9,3). Toutefois, le fait que dans le Ps 78 peste et flammes soient associées directement aux anges de malheurs et que la peste s'attaque aux «vies» des Égyptiens et non à leurs animaux, semble plutôt suggérer que Deber et Resheph, en tant qu'émissaires de la divinité, étaient compris comme puissances agissantes dans la version finale du psaume. À cet égard, l'hypothèse de Zenger, d'après lequel les versets $49 \mathrm{~b}-5$ oa sont une glose, est à considérer attentivement: dans ce cas, l'interpolation témoigne ainsi d'une compréhension démonique du passage encore à une époque tardive où l'insertion de l'expression « anges de malheurs » sert notamment à spécifier la nature de ces puissances ${ }^{17}$. Considéré sous cet angle, le Ps 78 atteste donc une tradition qui réinterprète le récit des plaies d'Égypte en les attribuant à l'action de puissances démoniaques, émissaires de la colère de Yhwh.

À cet égard, on rappellera que la forme plurielle des rəšāpîm n'est pas sans parallèles dans le Proche-Orient ancien. Elle est attestée en Égypte sur une inscription de Ramsès III à Medinet Habou, en contexte militaire, et à côté de Montou : ici les guerriers sur les chariots sont comparés à des Reshephs. Elle apparaît encore dans le Papyrus de Turin avec un contexte moins clair où il est question de se protéger des forces hostiles ${ }^{18}$. En outre, dans une liste de rituels accomplis par le roi à Ougarit, on fait mention des « Reshephs qui entrent dans

16 Il apparaît ailleurs seulement en Qoh 8,8 où il indique le congé militaire en temps de guerre.

17 Hossfeld et Zenger fondent leur idée d'une interpolation sur la présence du terme mišlahat qui est très rare et tardif (Hossfeld et Zenger 2011, p. 288-290). En revanche, il ne me paraît pas nécessaire de faire recours au messager de Yhwh qui punit l'Assyrie en Is 37,36 pour expliquer la présence des anges de malheur au verset 49 , comme le fait Lee 199o. Sur les raisons pour lesquelles il faut comprendre mal'âkim comme « anges » et non simplement comme messagers, voir infra, § 8.1.1.

18 Lipiński 2009, p. 220. Pour une traduction du texte de Médinet Habu, on peut voir Edgerton et Wilson 1936, p. 23-24; Le papyrus de Turin (Cat. 1982 = CGT 54O77) est encore inédit ; fac-similé et traduction en Pleyte e Rossi 1869-76, vol. 2, p. 122, pl. 85, II : 2. 
le palais royal $»^{19}$ : ici le pluriel fait très probablement référence aux statues de la divinité. Des Reshephs sont également connus au premier millénaire en Phénicie : l'inscription du roi Bod'ashtart dans le temple d'Eshmoun à Sidon, qui remonte au $\mathrm{V}^{\mathrm{e}}$ siècle av. n. è., contient l'expression 'rș ršpm, «terre des Reshephs $»^{20}$. Les attestations de Resheph en dehors de la Bible hébraïque nous amènent directement à la question plus générale de l'arrière-plan levantin et proche-oriental de ces puissances qui mérite une attention spécifique.

\subsection{Divinités proche-orientales et démons bibliques}

De manière générale, nous sommes mal renseignés sur la présence de Deber et Qețeb dans les panthéons du Proche Orient ancien. On sait qu'une divinité $\mathrm{Dbr}$ est connue à Ebla, car l'expression «Dabir, dieux d'Ebla » apparaît dans une liste d'offrandes ainsi que dans l'onomastique eblaïte ${ }^{21}$. Le mot est attesté en ougaritique où le toponyme poétique $a r s ̦ d b r$, «terre de pestilence», indique le royaume des morts ${ }^{22}$, mais pour le reste il s'agit d'un dieu mal connu. La présence de Qețeb au Levant est très incertaine ${ }^{23}$. En revanche, Resheph est une divinité ouest-sémitique très bien connue, associée à la guerre, à la peste et parfois au monde des morts. Son culte, bien étudié, est attesté sur une très longue période au Levant, en Égypte et dans toute la Méditerranée, depuis le $\mathrm{III}^{\mathrm{e}}$ millénaire jusqu'à l'époque hellénistique tardive (son nom est encore mentionné à Palmyre ${ }^{24}$. Il subit en outre des phénomènes d'assimilation avec d'autres divinités (Montou en Égypte et Apollon à Chypre). Son iconographie, bien établie, est celle d'un dieu combattant : en Égypte, il est représenté surtout

$19 K T U 1.91 .11$ (= RS 19.15.11).

$20 \quad$ KAI 15.

$21 \quad d d a-b i$-ir dingir ib-la ${ }^{k i}$ (Tм 75.G.1464, discuté par Pomponio et Xella, 1997, p. 123-124). Cette formulation semble, en outre, présupposer que Dabir était le dieu patron de la ville, même si les attestations sont très peu nombreuses pour une divinité poliade, comme le remarquent à juste titre Francesco Pomponio et Paolo Xella. Pour d'autres occurrences possibles du théonyme, mais non assurées, voir la discussion en Frey-Anthes 2007, p. 74; Blair 2009, p. 32-33.

$22 \quad K T U 1.5$. V $18 ; 1.5$. VI 6.

23 D'après Johannes de Moor (1988, p. 105) une possible attestation de Qețeb à Ougarit serait la forme $q z b$ en $K T U 1.5$ II 20-24, qui désignerait un attribut ou un agent du dieu Mot, mais le passage est très fragmentaire. Voir la discussion en Frey-Anthes 2007, p. 74-75; Blair 2009, p. 38-40.

24 Parmi les travaux monographiques, les plus importants sont ceux de Fulco 1976, Lipiński 2009 et Münnich 2013. Voir également Day 2000, p. 197-207. Pour les aspects iconographiques l'on peut se référer à Schulman 1979 et Cornelius 1994. Sur la réduction de Resheph à démon dans la Bible hébraïque, l'article de référence est Niehr 2003, suivi par Kató 2012 et Rudnig-Zelt 2015. Voir également Frey-Anthes 2007, p. 110-142; Blair 2009, p. 194-215. 
comme archer. Le fait qu'il soit le dieu responsable de la peste, et donc doué d'arc et de flèches, explique probablement son association avec Apollon. En Palestine, au Bronze Récent, ses attributs typiques sont le bouclier et une arme de poing ${ }^{25}$. Au premier millénaire, sa présence est documentée au Levant par une série d'inscription en Phénicie, en Cilicie, à Palmyre ainsi qu'à Chypre ${ }^{26}$.

Le statut de Resheph dans la Bible hébraïque a fait l'objet d'un certain nombre de discussions. La majorité des chercheurs interprètent son rôle affaibli dans les textes bibliques comme résultant d'un processus de « dédivinisation $»^{27}$. Il est en effet indéniable que Resheph apparaît dans la Bible hébraïque comme une puissance qui ne fait pas l'objet d'un culte et dont le pouvoir est entièrement subordonné à celui de Yhwh. Son domaine d'action, limité à la maladie, est beaucoup plus réduit non seulement par rapport à son statut au Levant à l'âge du Bronze (par exemple à Ougarit), mais également par rapport à d'autres contextes religieux de l'âge du Fer : il suffira, à cet égard, de penser à Chypre ou au royaume araméen de Samal où Resheph est l'une des divinités principales de la ville ${ }^{28}$. Dans la mesure où, dans la Bible hébraïque, Resheph agit exclusivement comme bras armé de Yhwh, il subit un processus de « démonisation ». Il faut, toutefois, observer que cette démonisation ne correspond pas à une véritable «démythologisation» car les contextes où il est nommé sont, au contraire, parmi les plus mythiques de la Bible hébraïque. À cet égard, l'idée de Maciej Münnich, d'après laquelle la réduction de Resheph à un démon serait intentionnellement motivée par la volonté d'éliminer des éléments païens mythologisant très anciens, est problématique ${ }^{29}$. En revanche, l'idée de Herbert Niehr qui attribue la «renaissance» de cette divinité en Palestine à l'infiltration phénicienne dans la région au cours du $\mathrm{v}^{\mathrm{e}}$ siècle mérite d'être discutée ${ }^{30}$. Niehr inscrit les processus de «démonisation» de Resheph

25 Il est représenté ainsi sur des statuettes retrouvées à Lachish et Megiddo : voir Keel et Uehlinger 2001, fig. 57,85 a.

26 Elles sont établies sous forme de liste et analysées par Münnich 2013, p. 209-214, 240-26o.

27 Frey-Anthes parle de «de-potenziert Gott», de même que Rudnig-Zelt 2015.

28 Le dossier chypriote du I ${ }^{\mathrm{er}}$ millénaire comprend une dizaine d'inscriptions, analysées par William Fulco (1976, p. 46-54), Edward Lipiński (2009, p. 229-236) et Maciej Münnich (2013, p. 247-256), et quelques théophores (ršpytn et 'bdršp, sur lesquels on peut voir Münnich 2013 (p. 249-250). Le culte de Resheph à Samal est attesté par l'inscription du roi Panamuwa I sur la base d'une statue représentant le dieu Hadad. L'inscription mentionne à plusieurs reprises Hadad, El, Resheph, Rakib El et Shamash comme garants du pouvoir royal $($ KAI $214=$ Gibson 1975, p. 6o-76, no. 13, voir notamment la formulation à la ligne 3 : wqm 'my ršs, « et Resheph s'est levé avec moi »).

29 Münnich 2013, p. 235-236.

30 Niehr 2003, p. 99-103. Cette infiltration serait, entre autres, soutenue par la fondation sur la côte de la ville d'Apollonia, contrôlée par Sidon. 
dans le contexte de changements plus profonds qui affectent les religions du Levant pendant cette période. Ces changements impliquent, entre autres, le développement d'un rapport plus personnel avec la divinité poliade. Le dieu patron de la cité est alors de plus en plus perçu comme protecteur de l'individu, tant dans le culte civique traditionnel que dans l'expression de la piété religieuse privée. On pourrait, en outre, rattacher à ce contexte la représentation de Yhwh comme «médecin », attestée dans plusieurs textes bibliques ${ }^{31}$. La démonisation de Resheph viendrait renforcer cette vision positive de la divinité alors allégée de ses côtés les plus obscurs.

Il ne faut cependant pas oublier que la question du prétendu « côté obscur » de Yhwh demeure complexe. En effet, la présence de messagers qui exécutent les ordres de la divinité ne la libèrent pas forcement de ses aspects négatifs et inquiétants : la représentation d'une divinité « transcendante $»^{32}$ qui envoie la guérison ou la maladie de loin, c'est-à-dire par ses messagers, coexiste avec des traditions qui attribuent des traits démoniaques à Yhwh lui-même. Dans un texte fameux de l'Exode (4,24-26) qu'on a longtemps jugé comme un résidu d'archaïsme mais qui pourrait avoir été rédigé plus tard que ce que l'on pense, Yhwh est décrit comme un démon qui attaque Moïse pour le faire mourir ${ }^{33}$. En outre, dans plusieurs passages qui nous intéressent ici, Yhwh reste le responsable ultime des actions de ses émissaires. Cette responsabilité est encore soulignée par des textes tardifs comme Is 54,16: « c'est moi qui ai créé le destructeur (mašḥit) pour défaire! ».

De manière générale, même si le degré d'autonomie qui leur est attribué est variable, l'action de ces puissances ainsi que la durée des fléaux restent largement contrôlées par la divinité elle-même. On peut comparer, à cet égard, les récits de $2 \mathrm{~S} 24$ avec son parallèle en $1 \mathrm{Ch} 21$ où l'ange exterminateur de Yhwh prend son initiative en autonomie ${ }^{34}$. Dans le Ps 91, Deber et Qețeb agissent également comme deux puissances autonomes. La perception de ces puissances comme des démons à part entière, et, de plus, dans des textes bibliques relativement tardifs, a parfois posé problème à l'exégèse. À cet égard, Niehr interprète le Ps 91 comme relevant d'un «paganisme littéraire ». Dans sa perspective, le recours à des éléments mythologisants «païens »- à savoir anciens et, donc, non-monothéistes - dont ce psaume est un exemple, ne reflèterait pas nécessairement de véritables croyances. En revanche, il

\footnotetext{
$31 \quad$ Niehr 1991.

32 Niehr 1991, p. 12-15.

33 Pour une proposition de datation de ce passage à l'époque perse, voir Römer 1994; pour sa réception ancienne, Goosmann 1993. Sur le geste apotropaïque de Zipporah, voir, plus récemment, et, entre autres, Hüllstrung 2003 ; Hays 2007. 
s'agirait d'une stratégie scribale qui, en réactivant et en exploitant des scénarios mythologiques en contexte poétique, permettrait d'affirmer de manière efficace la puissance absolue de $\mathrm{Yhwh}^{35}$. Cette théorie, souvent reprise par la recherche, se heurte néanmoins à quelques difficultés dans mon cas d'étude. Premièrement, ces puissances ne sont pas exclusivement attestées en poésie, mais apparaissent également dans quelques contextes de prose, tels que l'Exode, les livres de Samuel et les Chroniques où elles s'avèrent difficilement interprétables comme outils purement poétiques. Deuxièmement, si dans le cas de Resheph un arrière-plan levantin est assuré, ce même arrière-plan ne se reconstruit pas aussi aisément pour les autres puissances, rendant difficile l'évaluation de ces éléments mythologisants comme véritablement anciens. Dernièrement, et de manière plus générale, une compréhension exclusivement littéraire des passages poétiques ainsi que des procédés métaphoriques sous-jacents ne tient pas suffisamment compte des contextes d'usages de ces textes, notamment des Psaumes (y compris d'Hab 3, qui a la forme d'un psaume et, à certains égards, de Deut 32). Cette dernière remarque est d'autant plus pertinente dans le cas du Ps 91, pour lequel un nombre de témoignages important confirme son usage apotropaïque dès l'époque du Second Temple, dans les versions hébraïque et grecque. C'est donc vers ce contexte qu'il nous faut à présent tourner notre attention et, plus particulièrement, vers la traduction grecque de ces passages.

\section{Repenser les démons dans la LXX}

Le scénario des agents de destruction et de malheur tel qu'il apparaît dans la LXX présente des différences substantielles par rapport à la Bible hébraïque. Ces différences relèvent pour la plupart de l'activité des traducteurs qui ne semblent pas avoir toujours reconnu la nature de ces puissances. Dans le texte grec leur nom est parfois omis, parfois substitué par leur fonction, ou leur fonction est modifiée. L'exemple le plus évident et le mieux étudié est encore celui de Resheph, notamment car il s'agit de la puissance divine la mieux attestée au Levant. Dans la LXX de Deutéronome 32 son nom est traduit par «oiseaux » (öpveı) :

Niehr 199o, p. 210-220. 


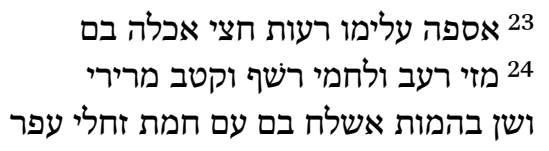

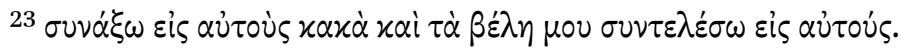

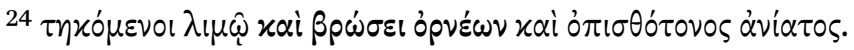

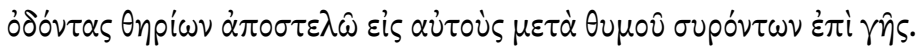

${ }^{23} \mathrm{Je}$ rassemblerai contre eux des malheurs et jépuiserai contre eux mes flèches.

${ }^{24}$ Consommés par la faim, en pâture aux oiseaux et une convulsion incurable!

J'enverrai contre eux les dents des bêtes sauvages avec le courroux de ceux qui rampent sur le sol.

Le contexte et la présence de $\beta p \omega \hat{\sigma} \iota \zeta$ semblent indiquer qu'il s'agit ici d'oiseaux de proie ou charognards, qui dévorent les corps de ceux qui sont « consommés par la faim » $(\tau \eta \varkappa o ́ \mu \varepsilon v o เ ~ \lambda \iota \mu \hat{\omega})$. Le verset ferait donc référence à une situation d'épidémie ou de famine, s'accompagnant de l'envoi d'une maladie (ỏ $\pi \bullet \sigma 0$ ó $\tau$ vos dंviatos) sur laquelle je reviendrai plus tard.

La situation est différente dans le cas d'Hab 3,5. Dans le texte retenu par Rahlfs et Ziegler, qui correspond aux codices Alexandrinus (A) et Marchalianus $(\mathrm{Q})$, Resheph n'a pas été traduit. Le verset 5 contient l'expression « ses pieds

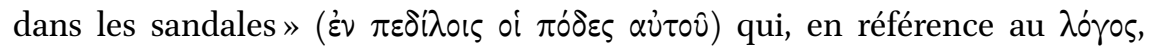
traduit Deber :

\section{כ3לוה מתימן יבוא וקדוש מהר פארן סלה

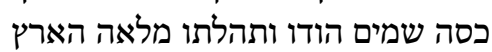 \\ 4

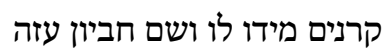

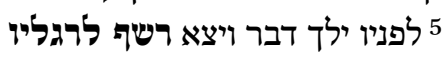

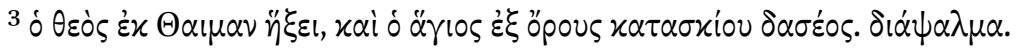

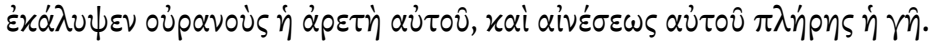

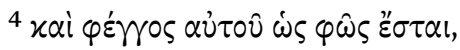

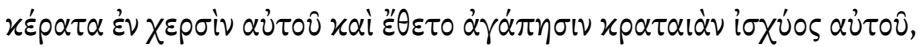

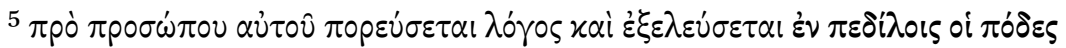

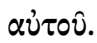


${ }^{3}$ Dieu viendra de Thaiman et le saint de la montagne ombreuse, épaisse. (Diapsalma).

Sa valeur a recouvert les cieux et la terre est pleine de sa louange. ${ }^{4}$ Son éclat sera comme la lumière, des cornes dans ses mains, il a établi un amour puissant de sa force, ${ }^{5}$ devant sa face s'avancera la parole et elle sortira, les pieds dans les sandales.

Le traducteur a introduit une série de choix exégétiques dans ces versets. Un premier exemple est la compréhension de Paran comme une montagne rem-

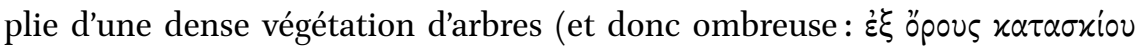

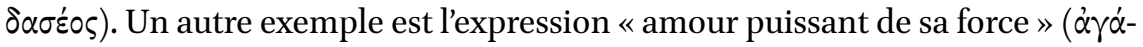

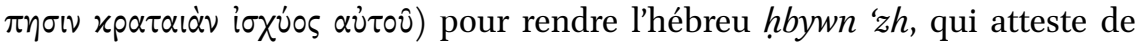
l'interprétation des cornes comme signes de puissance divine et masculine ${ }^{36}$. Dans ce contexte, on comprend mal, néanmoins, la relation घंv $\pi \varepsilon \delta i ́ \lambda$ oı et $r \check{s} p$, au point que le texte est marqué par une crux par Joseph Ziegler. Une ancienne hypothèse proposée par Charles Clermont-Ganneau et reprise par Henry Thackeray voyait dans ces sandales une référence à Persée qui serait à son tour une interprétation grecque de Resheph via Apollon : elle s'appuie sur des arguments spéculatifs et a été aujourd'hui abandonnée ${ }^{37}$. La solution est, en revanche, probablement à rechercher dans la tradition manuscrite du verset 5 b qui présente un certain nombre de divergences textuelles.

Le Vaticanus (B), le Sinaiticus (S) ${ }^{38}$ ainsi qu'une partie de la tradition indi-

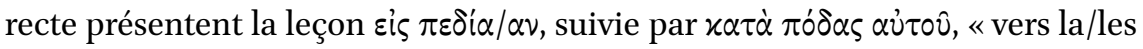
plaine/s, sur ses pas », alors que la tradition lucianique (L) présente la forme

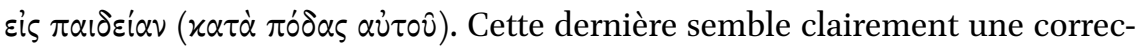

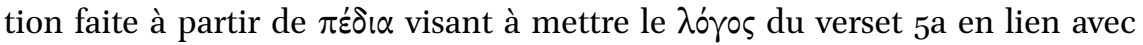
l'éducation. Le Papyrus Washington $(\mathrm{W})$, qui remonte au $\mathrm{III}^{\mathrm{e}}$ siècle de l'ère,

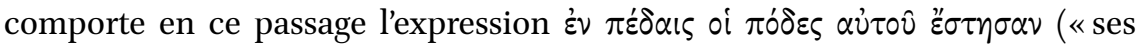
pieds étaient en chaînes »). Oi $\pi \delta ́ \delta \varepsilon \varsigma$ est ici sujet du verbe du verset 6 (हैं $\tau \eta \sigma \alpha \nu)$, qui dans la plupart des autres manuscrits est au singulier et se réfère à Yhwh, comme c'est le cas en hébreu ( corrigé au-dessus par $\pi \varepsilon \tau \eta$ - (avec effacement du $\dot{\varepsilon} v$ initial), et au-dessous par $\varepsilon \nu \pi \varepsilon \delta i \lambda \omega^{39}$.

$36 \quad$ Voir, à ce sujet, les remarques de Mulroney 2016, p. 121.

37 Clermont-Ganneau 1876, p. 374-376 ; Thackeray 1921, p. 51-54. Pour une critique convaincante de cette hypothèse, voir Harper 2015, p. 151-153.

38 Sur les accords entre B et S dans la LXX des Douze, voir Ziegler 1943, p. 37.

39 Le papyrus (W d'après Ziegler 1943, p. 8 et p. 32-34), faisant partie de la collection Freer, préserve des fragments des Douze petits prophètes. Pour l'édition, voir Schimdt et Sander 
Comme l'ont suggéré Cécile Dogniez, Joshua Harper et d'autres ${ }^{40}$, la forme $\varepsilon i \varsigma \pi \varepsilon \delta$ í $\alpha$ que nous trouvons dans B-S pourrait bien relever d'une lecture de la séquence $r s ̌ p ~ l r g l w$ comme $b s ̌ p l r g l w$, avec la confusion, fréquente, entre $r$ et $b$. La racine $s ̌ p l$, «être en bas », explique le choix de $\varepsilon i \varsigma ~ \pi \varepsilon \delta \dot{\prime} \alpha$ comme lecture de la Septante ancienne, sur la base de l'équivalence entre $\pi \varepsilon \delta i o v$ et špl/šplh, «terre basse» et donc «plaine», attestée également ailleurs ${ }^{41}$. La leçon du

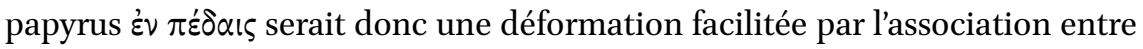

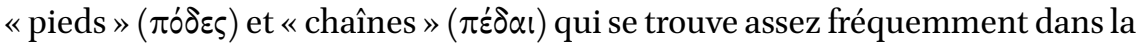

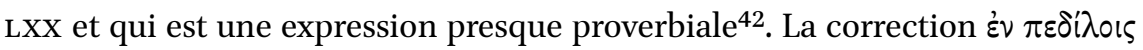
préservée par $A$ et $Q$ pourrait aller dans le même sens. Comme on le verra dans

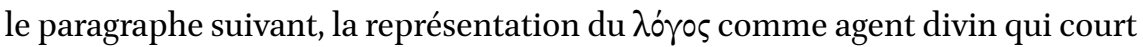
sur la terre est attestée ailleurs dans la Septante ${ }^{43}$.

Une telle variation au niveau de la tradition manuscrite indique que les traducteurs ne connaissaient certainement plus le nom de Resheph. Toutefois, cette conclusion peut être nuancée si l'on considère le témoignage de la version dite Barberini, une traduction du Psaume d'Habacuc réalisée entre le $\mathrm{I}^{\mathrm{er}}$ et le $\mathrm{III}^{\mathrm{e}}$ siècle de notre ère, probablement en Égypte. Cette traduction, dont la nature littéraire est très marquée, est préservée par quatre manuscrits. On la considère d'habitude comme une traduction indépendante ${ }^{44}$ car elle présente peu d'affinités avec les autres versions grecques connues. Dans le verset 5 elle lit notamment:

1927 qui considèrent ces corrections comme étant de troisième main et postérieures d'environ un siècle. Sur la nature du texte grec des Douze préservé dans ce papyrus, on peut voir De Troyer 2006.

40 Dogniez et al. 1999, p. 289-29o ; Harper 2015, p. 151-155; et Mulroney 2016, p. 217. Voir notamment Harper pour la reconstruction du verset 5 de la Septante ancienne.

41 Deut 1,7 ; Jos 11,$2 ; 12,8$. L'usage du pluriel $(\pi \varepsilon \delta i \alpha)$ pour traduire une forme hébraïque au singulier est attesté ailleurs dans les Douze (Joel 1,10;2,22). En revanche, l'idée de Dogniez selon laquelle la version du papyrus préserverait la Septante ancienne présuppose que la même erreur de lecture ( $b$ pour $r$ ) ait été commise de manière indépendante par au moins deux scribes, ce qui parait difficile. La version de la Septante ancienne serait alors

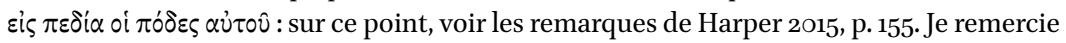
Jan Rückl pour son aide concernant la critique textuelle de ce verset.

$422 \mathrm{~S}_{3,34 ; 3}$ Mac 4,9; Ps 104,18; Sir 6,24; 21,19.

43 Voir infra, § 4.3.

44 Parmi les études principales sur la version Barberini on peut voir Thackeray 1911; Mercati 1955; Good 1959; Fernández Marcos 1976 (republié en allemand dans Fabry et Böhler 2007, p. 151-180); Fabry 2010; Dogniez 2012; et récemment la monographie de Harper 2015. D'après Natalio Fernández Marcos, cette traduction pourrait être attribuée à une «école de Symmaque», sur laquelle nous sommes, par ailleurs, mal informés. En revanche, les arguments avancés par Edwin Good, Heinz-Joseph Fabry et Joshua Harper pour une origine égyptienne me paraissent bien fondés. 


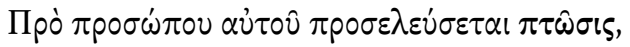

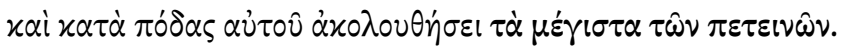

Devant lui ruine sortira,

et sur ses pas le plus majestueux des ailés suivra.

Le scribe responsable de cette traduction a reconnu en Deber, sinon une véritable maladie, du moins une puissance liée à la dévastation et il a traduit Resheph par un adjectif superlatif au neutre pluriel, donc à la lettre «les plus grands des êtres ailés ». Mais une traduction au singulier est également pos-

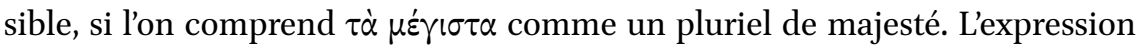
peut se référer à un ou plusieurs oiseaux ou, autrement, à un être divin doué d'ailes. Il faut, en outre, remarquer que cette traduction est similaire aux choix proposés par les réviseurs, également attestés dans la tradition manuscrite de Symmaque (őpveov) ainsi que chez Jérôme ${ }^{45}$.

Toutefois, bien que cela ait parfois été soutenu ${ }^{46}$, l'existence d'une tradition exégétique qui interprète Resheph comme un être ailé n'est pas nécessairement une innovation du judaïsme alexandrin; elle remonte probablement déjà à la Bible hébraïque ${ }^{47}$. Cette lecture est attestée particulièrement dans les traditions sapientielles. D'après Job 5,7, « les fils de Resheph volent très haut »:

\section{כי אדם לעמל יולד ובני רשף יגביהו עוף}

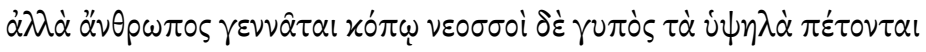

Il s'agit là très probablement d'une expression proverbiale qui n'a de sens que si l'on comprend les «fils de Resheph » comme des oiseaux, où l'hébreu ben a le sens de «membre d'un groupe » ou d'une classe ${ }^{48}$. Cette signification est bien préservée par la LXX de Job qui traduit « les petits des vautours » et rend ainsi explicite le lien intertextuel entre ce passage et Job 39,27 («c'est sur ton ordre

45 Alors que öpveov est attesté par la minuscule hexaplaire 86 (Ziegler 1943), les autres versions des réviseurs ont été préservées seulement par la tradition latine reflétée dans le commentaire de Jérôme: «Ubi diximus: Egredietur diabolus ante pedes eius [...] Aquila pro diabolo transtulit volatile; Symmachus autem et Theodotion et Quinta editio volucrem, quod Hebraice dicitur Reseph » (Jer., Comm. in Abacuc 2.3, p. 64O-641 [Migne PL 25, col. 1314]).

46 Lipiński 1999, suivi par Münnich 2013, p. 234-235.

47 Pour la réception de Resheph dans la LXx, voir l'étude fondamentale de Dogniez 2003.

48 Il ne me semble pas envisageable d'attribuer à bny ršp ici la signification de «flèches », pace Lipiński (1999, 2009, p. 26o-261), d'autant plus que la racine $g b h$, «voler», fait encore référence au vautour en Job 39,27. 
que l'aigle s'élève et que le vautour [rú $\psi$ ] passe la nuit près de ses petits »). En outre, dans un passage du Siracide préservé en grec et en hébreu, il est dit que «Yhwh fait voler la neige comme des oiseaux ( (texte de Masada) ou que «Yhwh répand la neige comme des oiseaux qui se posent» (texte grec et version du Caire $)^{49}$.

On peut vraisemblablement imaginer que cette interprétation juive de Resheph-oiseau était connue et exploitée par le traducteur du Deutéronome, car elle s'adaptait parfaitement au contexte de son passage biblique où il est question d'être dévoré. Devenir la proie des oiseaux est en effet l'une des punitions divines les plus courantes dans les malédictions proche-orientales. Ce châtiment est d'ailleurs déjà mentionné parmi les malédictions du Deutéronome ${ }^{50}$ et, de plus, se trouve parfaitement adapté pour un public de langue et de culture grecques qui connaît depuis Homère le déshonneur associé à ce type de mort. L'origine de cette interprétation demeure, par contre, problématique car les ailes ne semblent pas être un attribut de Resheph ni dans les textes ni dans l'iconographie du Proche Orient antique dont nous avons connaissance ${ }^{51}$. Toutefois, quelques hypothèses pourraient être formulées.

Cette association pourrait remonter au contexte phénicien et être, ainsi, antérieure à la Bible hébraïque. L'hypothèse pourrait être appuyée par une inscription bilingue phénicien-louvite datant du viI ${ }^{\mathrm{e}}$ siècle av. n. è., commandée par le roi Azatiwada et retrouvée en trois copies à Karatepe, en Cilicie ${ }^{52}$.

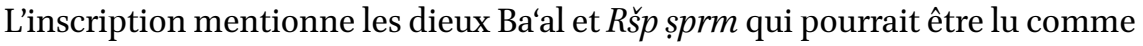
« Resheph des oiseaux», attestant ainsi d'une ancienne association de cette divinité avec les oiseaux. Toutefois, d'autres lectures de cette expression sont également possibles et ont été proposées par les chercheurs ${ }^{53}$.

Une deuxième interprétation, proposée par Lipiński et suivie par Niehr, voit dans la représentation de Resheph en oiseau une influence égyptienne du dieu à tête de faucon Montou-Horus. Cette hypothèse me paraît peu probable, premièrement car une équivalence directe entre Horus et Resheph n'est jamais attestée par les sources (au contraire de celle entre Resheph et Montou et de celle entre Montou et Horus) et serait donc à postuler indirectement. Deuxièmement, à l'époque hellénistique l'association entre Montou et Resheph est seulement attestée par deux inscriptions du temple de Karnak,

\footnotetext{
49 Sir $43,17=43,18 \mathrm{LXX}$.

50 Deut 28,26 .

$5^{1} \quad$ Voir, à ce sujet, Schulman 1979; Cornelius 1994, p. 253.

$52 \quad$ KAI 26, 10-11; Röllig 1999, Phu A/II, p. 52-53, qui ne traduit pas l'épithète.

53 Notamment «Reshephs des boucs », qui correspondrait mieux à son équivalent louvite (le dieu-bouc dénommé Runtiya, maître des animaux sauvages). Voir Xella 1999, p. 701702 ; et Münnich 2013, p. 210-211.
} 
commandées par Ptolémée III. La dédicace parle de «Resheph qui habite dans la demeure de Montou » ${ }^{4}$ : les deux divinités, puissances tutélaires de la guerre, sont donc rapprochées car elles partagent le même sanctuaire, mais elles restent, cependant, bien distinctes. En outre, la statue de Resheph représente le dieu en armes, sans ailes ni tête de faucon ${ }^{55}$.

En revanche, il pourrait s'agir d'une exégèse interne au Ps 91,5 où l'orant est terrorisé par Deber et Qețeb ainsi que par la « flèche qui vole pendant le jour ». Dans d'autres passages bibliques tels que Ps 76,4 , le nom de Resheph est compris comme «flèche », par association métaphorique entre flèches et flammes, les deux volant à travers le ciel. Il est encore question de flammes ardentes dans Cant 8,6. Il n'est pas invraisemblable d'imaginer que ces flèches volantes aient été perçues comme de véritables êtres ailés, surtout au vu des contextes métaphoriques dans laquelle l'expression est employée. Cette interprétation pourrait être renforcée par le fait que les ailes sont, dans la glyptique mésopotamienne et levantine, des attributs des êtres divins et surtout démoniaques ${ }^{56}$.

Par conséquent, faudrait-il voir dans la représentation de Resheph comme «le plus grand des ailés », un démon ailé de l'éclair, ensuite réduit à un oiseau, comme le proposait déjà André Caquot ${ }^{57}$ ? Cette lecture est certes séduisante dans le cas d'Hab 3 dans la version Barberini qui nous présenterait donc l'arrivée de Yhwh entouré d'une série de grandes puissances ailées. Toutefois, elle demeure problématique pour les autres passages qui semblent s'inscrire dans un contexte explicitement naturel et paysager et font plutôt penser à une exégèse originelle de Resheph comme « oiseau».

À cet égard, il faut néanmoins souligner que les êtres ailés mentionnés en Deut 32 ne doivent pas nécessairement être identiques à ceux du texte Barberini d'Hab 3. Une multitude de rapaces prêts à se jeter sur des cadavres ne semble pas constituer un complément approprié pour une théophanie divine qui insiste sur la puissance et la lumière émanant de Yhwh, ici représenté comme divinité solaire. Une hypothèse plus pertinente serait alors de voir dans le texte Barberini d'Hab 3,5 une interprétation de Resheph comme phénix. Oiseau mythologique associé à la lumière et au feu, de dimensions gigantesques, le

$54 \quad$ Sternberg-El Hotabi 1993, p. 110-111.

55 Cornelius 1994, no. 72 ; voir aussi la discussion en Münnich 2013, p. 221. L'hypothèse de Lipiński (2009, p. 255-257) se fonde sur une série d'équivalences indirectes qui voient, d'un côté, Montou assimilé à Resheph et à Apollon, d'un autre côté, Apollon identifié à la fois à Resheph et à Horus, et, enfin, l'association entre Horus et Montou. La combinaison de ces associations permettrait donc de postuler une identification entre Resheph et Horus, qui n'est cependant jamais attestée par les sources anciennes.

$5^{6}$ Voir, par exemple, Avigad et Sass 1997, no. 159 et 173.

57 Caquot 1956, p. 6o-61. 
phénix est à bien des égards «le plus majestueux des ailés ». En outre, il est étroitement associé au soleil et à la vision de la divinité dans d'autres traditions juives, comme celles du livre des secrets d'Hénoch (2 Hen 12) et de l'Apocalypse grecque de Baruch (3 Bar 6,2). De plus, les traditions sur le phénix s'expliqueraient bien dans le contexte égyptien d'où la version Barberini semble provenir $^{58}$. À ce propos, il faut également rappeler que les fragments de l'Exagogé du poète juif Ezéchiel dit «le Tragique », actif à Alexandrie au II ${ }^{\mathrm{e}}$ siècle av. n. è., nous transmettent la description d'un oiseau merveilleux de grande taille, traditionnellement identifié au phénix, qui est décrit comme le «roi de tous les oiseaux» et dont il est dit qu'il marche « soulevant rapide le pas du pied $»^{59}$.

Les témoignages ici analysés démontrent que, à côté des traditions grecques qui ignorent la nature de Resheph, comme celle attestée par la Septante ancienne d'Habacuc, d'autres en proposent une exégèse juive qui devait être courante à l'époque. Comme ceci avait déjà été suggéré par $D o g n i e z^{60}$, la réception de Resheph en oiseau adoptée par la Septante ancienne de Deut 32,24 et par le texte Barberini d'Hab 3,5 (avec ses variations «égyptianisantes», telles que la référence au phénix) ne serait pas une invention du traducteur, mais bien plutôt un thème qui lui était déjà connu ${ }^{61}$. Cette interprétation aura une certaine fortune dans le judaïsme ancien : adoptée par les réviseurs de la LXX, l'exégèse de Resheph en oiseau est également attestée par les Targums Onkelos et Pseudo-Jonathan en Deut 32,24, où la punition réservée aux Israélites est d'être dévorés par des rapaces et possédés par toute sorte de démons. Elle est encore mentionnée dans le Midrash Rabbah ${ }^{62}$. De manière générale, dans la tradition rabbinique, l'interprétation de Resheph en oiseau coexiste avec la tradition qui le considère comme un démon ${ }^{63}$. Une forme de conciliation entre ces deux traditions exégétiques est attestée par le traité Pesachim, dans une section qui établit la liste d'une série de noms de démons selon les lieux où ils résident ou se manifestent : « les démons qui se trouvent sur les toits sont

$5^{8}$ Cette interprétation avait déjà été suggérée par Fernández Marcos (1976, p. 16, note 42), sans pourtant trouver de véritable suite. Pour le phénix en Égypte, voir Vernus et Yoyotte 2005, p. 380-382. En revanche, le référence au phénix dans la LXX de Cant 8,6 proposée par Lipiński (1999, p. 255) ne me paraît pas suffisamment soutenue par le texte grec. Lautre référence au phénix signalée par Lipiński dans la LXX (Job 29,18) est également incertaine: dans ce contexte le mot $\varphi$ oiv $\xi_{\xi}$ pourrait bien faire référence à une palme, et non à l'oiseau. Vor, sur ce point, Angelini 2015, p. 41-43.

59 Fr. 17, pour texte et traduction française voir Lanfranchi 2006, p. 283-296.

6o Voir, notamment, Dogniez 2003.

61 Elle explique également, je crois, le choix de l'adjectif $\pi \varepsilon p i \pi \tau \varepsilon p \circ \varsigma$ («qui vole autour») dans la Lxx de Cant 8,6.

$62 \quad$ Ex.R. 12.

63 b.Ber. 5 a. 


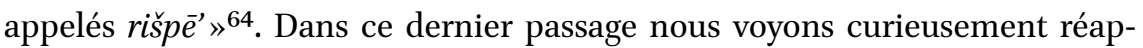
paraître des rəšāpîm au pluriel. Leur nature a été cependant profondément resémantisée par rapport aux divinités plurielles dont il était question dans les textes ougaritiques et égyptiens. À Ougarit, les différentes manifestations du dieu, matérialisées par la présence de plusieurs statues de la divinité, étaient rendues par le pluriel, correspondant alors à une manière d'exprimer sa puissance. Les dieux de type Resheph attestés dans le temple de Medinet Habu semblent tous avoir eu une même fonction militaire car ils servent de terme de comparaison pour exalter la force des soldats de Ramsès III. Dans le passage du Talmud, en revanche, le pluriel souligne qu'il s'agit d'une multitude d'entités dont la nature est désormais indéfinie et mal connue, qu'ils soient démons ou oiseaux.

\section{Le $\lambda$ óros comme agent démoniaque}

Le deuxième agent de malheur qui intervient à la fois dans le Ps 91 et dans le texte d'Habacuc est Deber. Les traducteurs ont lu dans les deux cas deber comme $d \bar{a} b \bar{a} r$ et ils en donnent deux traductions différentes selon les deux sens du mot hébreu : $\pi \rho \alpha ́ \gamma \mu \alpha$ dans le Ps 90 (= $91 \mathrm{TM}$ ), sur lequel je reviendrai plus tard, et $\lambda$ óros en Hab 3,5. Cette deuxième solution nous présente l'image du $\lambda$ ó os comme une puissance qui marche devant Yhwh, à la tête du cortège divin : il s'agit du seul personnage qui émerge dans la théophanie outre la divinité (dorénavant Logos). Comme on l'a vu, cette représentation varie selon les différentes traditions manuscrites :

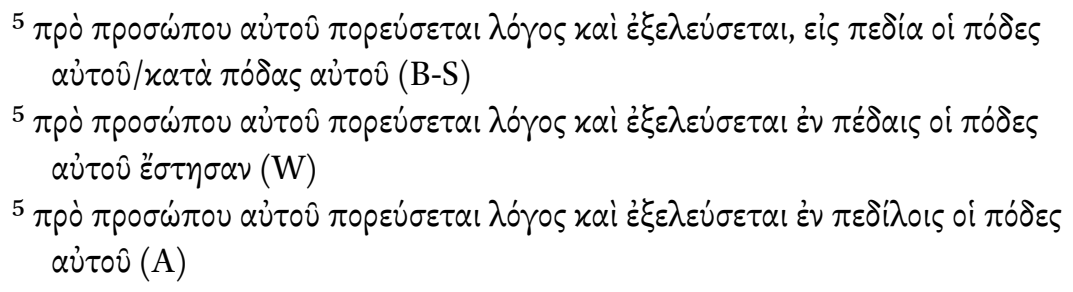

Les réviseurs proposent, quant à eux, des termes qui font référence au domaine

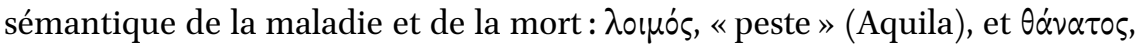

64 b.Pesah. 111 b. 
«mort» (Symmaque et une partie de la tradition hexaplaire) ${ }^{65}$. La version Barberini choisit $\pi \tau \hat{\sigma} \sigma \iota \varsigma$, «chute», «ruine», mais également «calamité». Bien que ces traductions soient plus proches de la signification littérale de l'hébreu, aucune d'entre elles ne semble véritablement refléter la nature de Deber comme puissance démoniaque. Une telle méconnaissance s'inscrit dans le cadre plus large des traditions rabbiniques et chrétiennes dans lesquelles la nature démoniaque de Deber semble avoir été définitivement oblitérée, contrairement à ce qui arrive dans les cas de Resheph et Qețeb.

En revanche, la représentation du Logos préservée par les manuscrits de la Septante et accueillie par Théodotion (du moins si l'on fait confiance au témoignage de Jérôme ${ }^{66}$ ) mérite quelques réflexions supplémentaires. Alors que la version du papyrus $(\mathrm{W})$ souligne le rapport de subordination entre la divinité et le Logos, les onciaux (B-S et A) mettent l'accent sur son dynamisme.

Il faut se retenir de la tentation de voir dans ce passage une lecture spiritualisante ou trop abstraite de la théophanie. Tout d'abord, l'idée de la parole divine (dābār $)$ comme puissance céleste et instrument de l'action de Yhwh est déjà présente dans la Bible hébraïque, notamment dans les traditions qui font référence à son rôle actif à côté de la divinité lors de la création. Ce rôle est souligné dans le Ps 33,6 (= 32,6 LXx): «les cieux ont été faits par la parole de Yhwh, toute leur armée par le souffle de sa bouche »; et dans le Ps 103,19-20 où le statut de la parole de Yhwh est clairement supérieur à celui des autres messagers de la cour céleste ${ }^{67}$ :

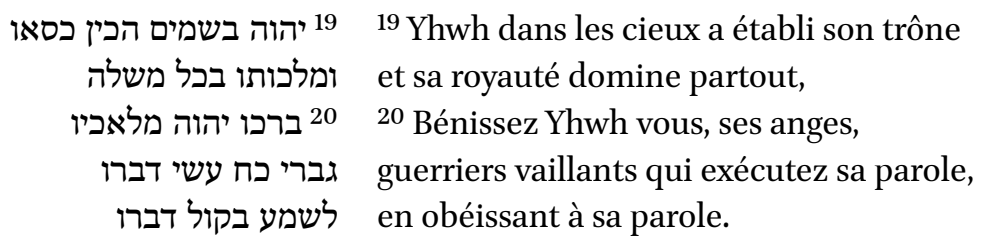

Comme Dogniez l'a déjà observé68, l'image de la parole qui court sur la terre est également développée dans des textes tardifs de la Bible hébraïque, tel que Is $55,10-11$ :

\footnotetext{
65 Ziegler 1943, apparatus II.

66 Jer., Comm. in Abacuc 2.3, 641 [Migne PL 25, col. 1314].

67 Mais comparer aussi Ps 107,19-20 (= 106,19-20 LXX), où Yhwh guérit par sa parole, et Ps $119,89(=118,89$ LXX $)$ où la parole est dressée dans les cieux.

Dogniez 2003, p. 551.
} 


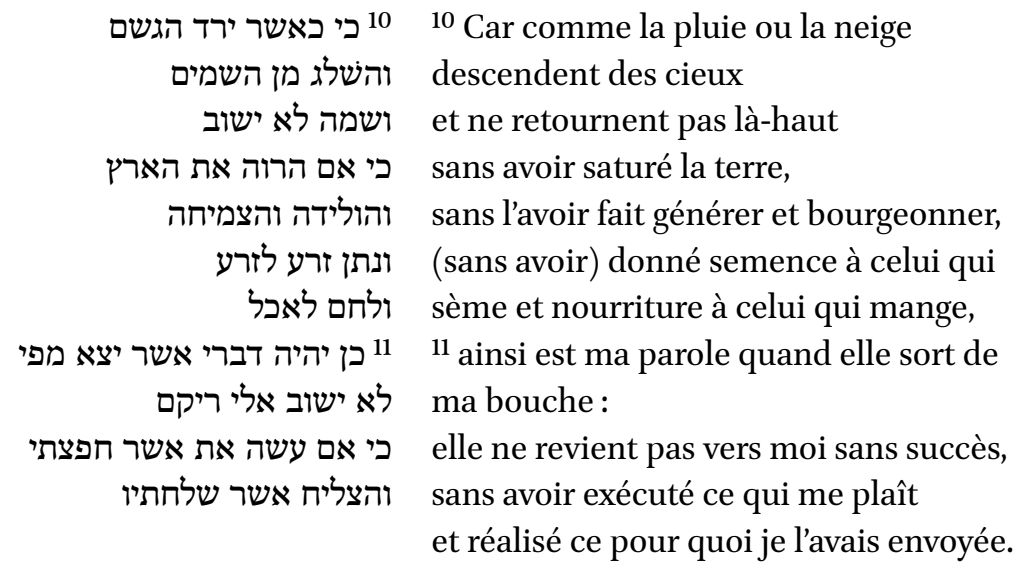

10 כי כאשר ירד הגשם

והשלג מן השמים

ושמה לא ישוב

והולידה והצמיחה

ונתן זרע לזרע ורידת

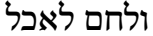

לא ישוב אלי ריקם יריה די אשר

Le Ps 147,15-18 est une variation de la même scène : «Il envoie ses ordres sur terre, si rapidement court sa parole. Il donne la neige comme la laine [...] Il envoie sa parole et (les) fait fondre, il fait souffler son vent, les eaux coulent ». La parole de Yhwh qui court, légère sur la terre, suivant les ordres de Yhwh, constitue donc un parallèle étroit au Logos qui s'élance sur les plaines de Yhwh en $\mathrm{Hab} 3,5$.

Ce motif est encore développé dans les traditions du judaïsme hellénistique, et notamment dans l'œuvre de Philon où le Logos est présenté à plusieurs reprises comme un intermédiaire de l'action divine, ainsi que le montrent plusieurs passages de son traité Quis est l'héritier des biens divins ? ${ }^{69}$ :

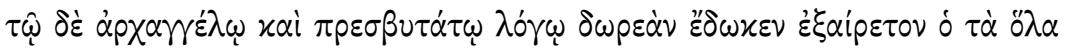

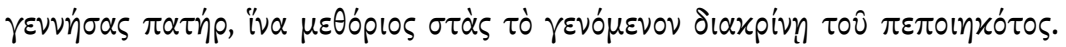

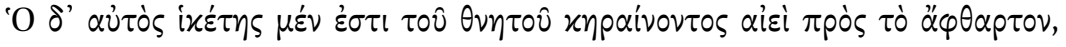

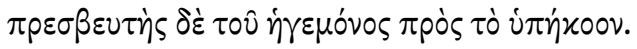

C’est au Chef des anges, au Logos très vénérable, que le Père, générateur de l'univers, accorda en faveur particulière de se tenir au milieu pour séparer le créé du créateur. Il est à la fois l'intercesseur du mortel toujours inquiet auprès de l'incorruptible et l'ambassadeur du souverain auprès du subordonné.

69 Heres 205 [trad. Harl, Paris, Cerf, 1966] ; voir également Heres 206-207, 225. Sur la rapidité du mouvement du Logos, voir aussi Cher. 28. Pour une analyse de ces passages, voir Calabi 2011. 
Une réception significative du Logos comme agent divin se trouve dans le livre de la Sagesse (Sap 18,15-18) où la «parole» envoyée par dieu «saute» du trône céleste dans le silence de la nuit pour tuer les nouveaux-nés des Égyptiens :

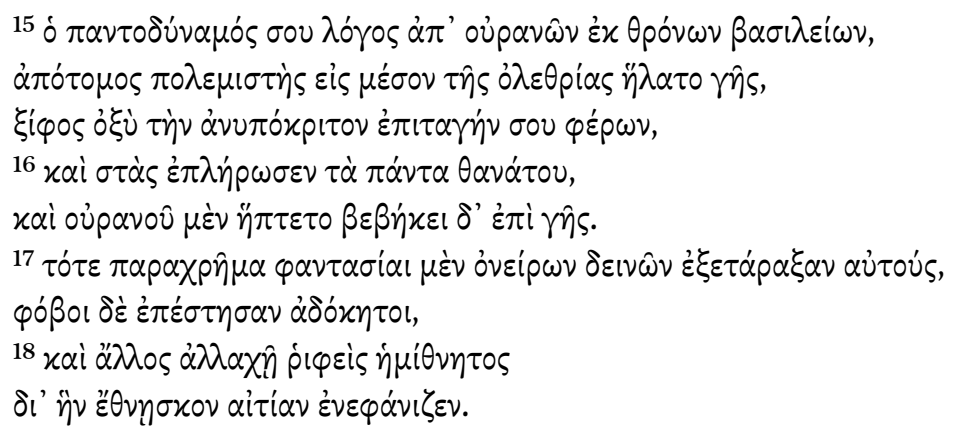

${ }^{15}$ Ta Parole toute puissante, depuis les cieux s'élança du trône royal, guerrier impitoyable, au milieu de la terre ruineuse, avec pour épée tranchante ton ordre irrévocable, 16 et en se dressant elle remplit tout de mort, elle touchait le ciel et foulait la terre.

${ }^{17}$ Aussitôt soudainement des visions d'horribles cauchemars les bouleversèrent, terreurs inattendues apparurent, 18 et chacun jeté d'un côté ou de l'autre, à moitié morts, ils manifestèrent la raison pour laquelle ils mouraient.

La parole reçoit une série d'épithètes qui la décrivent comme «douée de tous

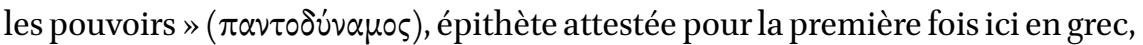

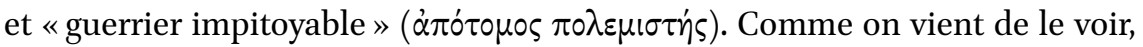
la représentation du Logos à côté du trône céleste a ses origines dans la Bible hébraïque. À cet égard, il est sans doute utile de rappeler que l'association du Logos avec l'activité démiurgique de la divinité n'est pas, à cette époque, une spécificité du judaïsme, mais qu'elle est largement développée dans les philosophies hellénistiques telles que le stoïcisme et le platonisme. C'est également un thème important pour les courants gnostiques et hermétiques, dont le Corpus Hermeticum nous offre les meilleurs exemples ${ }^{70}$. Dans ces contextes,

$70 \quad$ Notamment dans le Pimandre ( $\mathrm{CH} 1,1-11)$. Sur l'identification entre Logos et Hermès dans le Corpus Hermeticum, voir récemment Van den Kerchove 2012, p. 28-44 et bibliographie relative. 
la parole est souvent identifiée au dieu Hermès ${ }^{71}$ : ce dernier, en tant que puissance liée au langage et à la communication reçoit parfois lépithète de $\lambda \operatorname{ó}{ }_{10} \varsigma^{72}$. L'association entre Hermès et la parole est bien connue des auteurs chrétiens. Non seulement elle est citée dans les Actes de Luc (14,12), mais elle est également exploitée par les apologistes du $\mathrm{II}^{\mathrm{e}}$ siècle pour défendre leur propre identification entre le Logos et le Christ. D’après Justin, par exemple, l'idée d'un Logos fils de dieu n'a probablement rien de surprenant pour les Grecs, vu qu'Hermès lui-même est défini comme « Logos messager du dieu », à savoir de

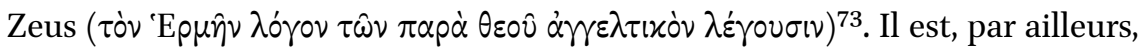
légitime de se demander si une telle association, qui demeure bien établie au moins jusqu'aux auteurs du $\mathrm{IV}^{\mathrm{e}}$ siècle $^{74}$, ne serait pas à l'origine du Logos en sandales attesté par une partie conséquente de la tradition manuscrite d'Hab 3,5 , les sandales ailées étant un attribut typique du dieu Hermès ${ }^{75}$.

Sur cette base, certains chercheurs ont voulu repérer des références plus ou moins directes à la notion grecque du Logos chez des auteurs juifs qui visaient davantage à se positionner par rapport aux traditions philosophiques de l'époque, tels que Philon ou l'auteur du livre de la Sagesse ${ }^{76}$. À ce propos, Giuseppe Scarpat voit dans l'épithète $\pi \alpha v \tau o \delta$ v́ $\alpha \mu \circ$ ( « tout-puissant ») attribuée à la parole en Sap 18,15 une référence polémique aux conceptions gnostiques

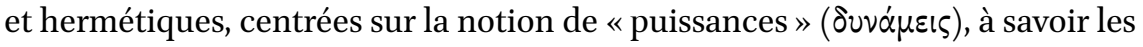

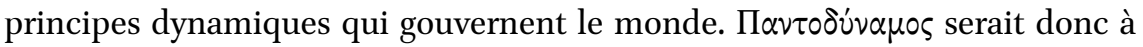
traduire plus précisément par «doué de toutes les puissances »77. Une représentation du Logos saint qui « saute» ( $\dot{\varepsilon} \kappa \pi \eta \delta \dot{\alpha} \omega)$ vers le monde (littéralement «la nature») se retrouve notamment dans le Pimandre, le premier traité du

71 Cette association est explicite pour la première fois chez Platon ( $\mathrm{Cra} .407 \mathrm{e})$; voir aussi Plut., Mor. $757 \mathrm{~b}$; Elien (NA 10, 29) dit que l'ibis est aimé par Hermès car « il est le père des $\lambda$ 'ó » ». La référence à l'ibis renvoie à l'association entre Hermès et Thot, dieu de la parole en Égypte qui est également une des figures protagonistes du Corpus Hermeticum.

Lucianus, Apol. 2, 6, Gall. 2, 23. Sur Hermès comme puissance de la communication, voir Bettini 200o, p. 5-51; Pisano 2014, p. 245-251.

73 Justin, Apol. 1, 22, 2. Voir également Apol. 1, 20-22. Voir aussi Clem., Strom. 6, 15, 132.

74 Iamb., Myst. I, 1; Synes., Ep. 101, 68. Eun., vs 10, 5, 5.

75 Il est utile, à cet égard, de mentionner un passage de la Réfutation des hérésies d'Hippolyte de Rome qui cite une exégèse allégorique et, par ailleurs, inconnue d'une œuvre astronomique attribuée à Aratos de Soles. Ce passage identifie le Logos non pas à Hermès, mais à Persée, qui avait emprunté les sandales ailées au dieu : «Persée est le Logos, fils ailé de Zeus» (Hipp., Haer. 4, 49). Bien que certains chercheurs pensent que l'œuvre d'Aratos puisse s'inspirer d'une source gnostique, l'existence d'une telle source n'est pas assurée. Voir Scholten 1991, p. 515-516.

76 Voir, notamment, Radice 2011 qui souligne l'influence des traditions allégoriques sur Philon.

Scarpat 1999, p. 264-270. 
Corpus Hermeticum ${ }^{78}$. En s'en tenant au témoignage d'Irénée, David Winston souligne, pour sa part, que les écrits gnostiques décrivent la Sagesse qui bondit directement de la divinité 79 .

Il faut toutefois souligner que l'insistance sur l'omnipotence de la parole divine a son parallèle le plus étroit au sein même du livre de la Sagesse.

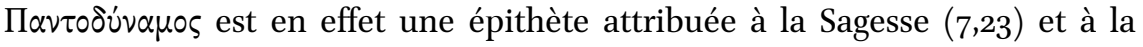
divinité $(11,17)$, à savoir les deux véritables puissances agissantes du livre ${ }^{80}$. Cette épithète établit donc un lien entre le Logos, la Sagesse et la divinité, en

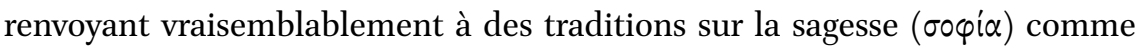
agent de la cour céleste qui précède la création et qui en supervise le travail avec la divinité. Il s'agit d'un thème important dans les traditions sapientielles de langues hébraïque et grecque, dont l'exemple le plus fameux est celui de Prov 8,22-31. En Sir 24, conservé exclusivement en grec, la Sagesse parcourt toute la terre avant de s'établir sur Israël. En outre, plusieurs passages dans la LXX associent étroitement $\lambda o_{\gamma}$ ○s et $\sigma \circ \varphi^{i} \alpha^{81}$.

Toutefois, la description du Logos en Sap 18,15-25 a une particularité qui la différencie des passages mentionnés auparavant. Le Logos est ici représenté non comme un partenaire de la création, mais comme un agent de destruction : il assume le rôle qui était propre au mašhît dans le livre de l'Exode. Il faut, à cet égard, souligner que la nature d'entité destructrice et malfaisante du mašhît est également bien développée dans la traduction grecque, non seulement en Ex 12, mais également en $1 \mathrm{Ch}$ 21, dans l'épisode de la peste envoyée par Yhwh. En Ex 12,23, l'envoyé de Yhwh qui sème la mort parmi les Égyptiens est traduit par $\tau \dot{v} v \dot{\partial} \lambda \varepsilon \theta p \varepsilon \dot{o v} \tau \alpha$, «le destructeur». Cette forme est un calque sémantique de l'hébreu car il s'agit d'un participe dérivé d'une racine « ruiner », « détruire » ( $\partial \lambda \nu \mu l)$, dont l'adjectif $\partial \lambda \varepsilon \dot{\varepsilon} \theta$ plos, « ruineux », et le substantif $\partial \lambda \varepsilon \theta \rho \varepsilon \dot{\alpha} \alpha$, « ruine » sont fréquents en grec, bien que le verbe ( $\partial \lambda \varepsilon \theta \rho \varepsilon v i \omega)$ soit employé très rarement en dehors de la LXX. En 1 Paral 21,15 (= 1 Ch 21,15 TM), mašḩit est traduit par un

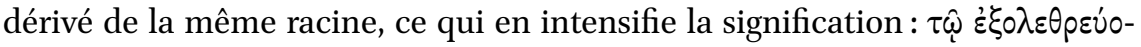
$\nu \tau \iota$, « celui qui détruit complètement». Ce participe semble donc se spécifier progressivement pour connoter des puissances destructrices. À cet égard, il

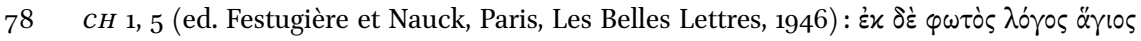

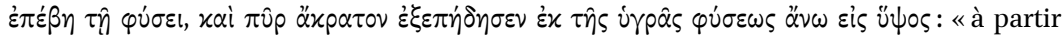
d'une lumière un Logos saint se dirigea sur la nature et un feu pur se dégagea vers le haut à partir de la nature humide ». Comparer, en outre, avec les versets 10-11 où le Logos s'élance pour s'unir au Nous démiurge.

79 Iren., Adv. Haer. 1, 23, 2 ; 1, 29, 2. Voir Winston 1979, p. 316.

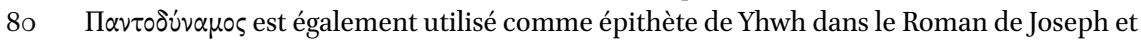
Aseneth $(11,9)$.

81 Par exemple Jer 8,9; Prov. 1,2 ; 5,1; Sir 4,24; 4 Mac 1,15. 
n'est pas sans intérêt de noter qu'on le retrouve mentionné dans une formule apotropaïque contenue dans un papyrus magique d'origine chrétienne, classé

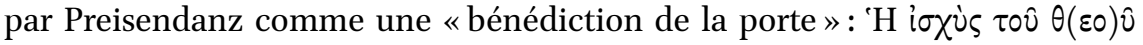

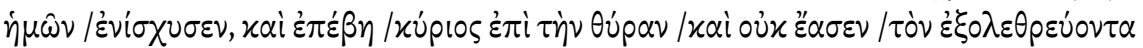
| $\dot{i \sigma \varepsilon \lambda \theta \varepsilon i ̂ \nu: ~ « ~ L a ~ f o r c e ~ d e ~ n o t r e ~ d i e u ~ a ~ p r i s ~ v i g u e u r, ~ e t ~ l e ~ S e i g n e u r ~ e s t ~ a r r i v e ́ ~ s u r ~ l a ~}$ porte, et il n'a pas laissé rentrer l'exterminateur ${ }^{82}$.

La description du Logos dans le chapitre 18 rappelle de près celle de l'ange qui amène la pestilence en $1 \mathrm{Ch} 21,16$, car les deux sont dotés d'une épée et occupent l'espace entre ciel et terre. En outre, dans la deuxième moitié du chapitre l'activité du Logos est mise en parallèle avec celle d'un autre agent de la colère de Yhwh, à savoir le fléau qui fait suite à la punition de Coré en Num 17 (versets 10-15). Alors que dans le livre des Nombres il est désigné génériquement comme «coup », «fléau » (negep, traduit en grec par $\theta p \alpha v 0$ livre de la Sagesse, il intervient comme un agent personnifié, qualifié au mas-

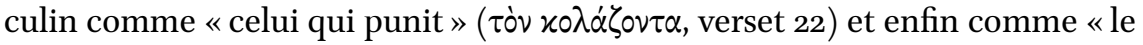
destructeur » (ó $\partial \lambda \varepsilon \theta \rho \varepsilon \dot{\omega} \omega \nu$, verset 25).

Ces remarques permettent de rapprocher la description du Logos en Sap 18,15-18 des représentations d'autres agents divins de punition qui, déjà présentes dans la Bible hébraïque, sont prolongées dans la LXX. Cependant, elles n'expliquent pas encore entièrement le caractère militaire attribué au Logos à la fois dans le livre d'Habacuc et dans la Sagesse. Il est utile, à ce sujet, d'observer que, d'un côté, ce Logos en armes ressemble aux divinités guerrières du monde classique telles qu'Apollon en bataille et surtout Athéna. D'après les étymologies anciennes, Athéna reçoit l'épithète de Pallas notamment car elle

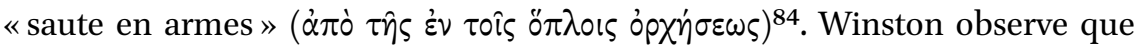
la même forme verbale est utilisée pour décrire le mouvement du Logos en Sap 18,15 ( de la tête de Zeus dans les Aitia de Callimaque ${ }^{85}$. D'un autre côté, la description du Logos en Sap 18 a un parallèle au chapitre 5 du livre, notamment aux versets 17-20, où la divinité invite sa création à participer à la punition des impies. Ce passage utilise un vocabulaire et un imaginaire militaire très proches de ceux du chapitre $18^{86}$. Un parallèle ultérieur se retrouve dans le texte grec d'Is

\footnotetext{
$82 \quad$ P 2 a (Preisendanz, vol. 2).

83 Num 17,12 et 14.

84 Plat., Cra.406d.

85 Call., fr. 37 Pfeiffer; Winston 1979, p. 317-318.

86 Sap 5,17-20: «Il prendra comme armure son zèle et il armera la création pour se défendre des ennemis. /Comme cuirasse, il revêtira la justice, comme casque, il mettra un jugement sincère. /Il prendra comme bouclier (sa) sainteté invincible,/ Il affûtera une colère inflexible en guise d'épée et l'univers viendra combattre avec lui contre les insensés ».
} 
$11,4 \mathrm{~b} \alpha$ où il est encore question d'une punition divine, qui tombe cette fois sur Israël. L’expression métaphorique de l'hébreu, והכה ארץ בשבט פיו il frappera le pays par sa bouche comme par un bâton»), est traduite par $\pi \alpha \tau \dot{\alpha} \xi \varepsilon ı ~ \gamma \hat{\eta} \nu \tau \hat{\omega}$

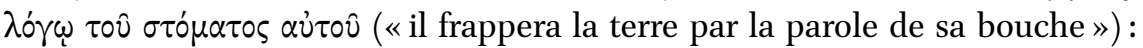
cette solution crée un parallèle parfait avec l'esprit ( $r u \hat{a} h / \pi v \varepsilon \hat{\mu} \mu \alpha)$ qui agit dans

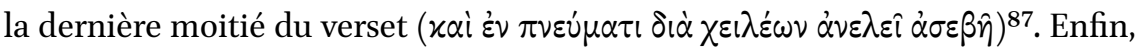
le Logos est nommé à plusieurs reprises comme agent de châtiment dans les Psaumes dits «de Salomon », composés autour de la moitié du I ${ }^{\mathrm{er}}$ siècle avant notre ère, probablement en hébreu, mais conservés seulement en grec et en syriaque. Dans le psaume 17, la « parole de la bouche » de Yhwh est préposée à la punition de la terre, qui ici indique l'humanité dans son entier, alors que la sagesse et la joie sont instruments de bénédiction ${ }^{88}$.

Plusieurs conclusions sont à tirer de cette analyse. Tout d'abord, il est raisonnable de voir à l'arrière-plan de la représentation de la Parole/Logos en Sap 18 une référence à des conceptions du Logos propres aux philosophies hellénistiques, dans la mesure où la démarche philosophique de l'auteur entre en dialogue avec les autres courants de l'époque. Cependant, la mesure dans laquelle cette référence doit être interprétée dans le sens d'une polémique directe est, à mon avis, moins claire. Le rôle du Logos au chapitre 18 doit davantage être mis en relation avec son rôle dans le reste du livre et, de manière plus large, dans les traditions sapientielles. En outre, la fonction du Logos en Hab 3 ainsi qu'en Sap 18 s'enracinent dans une tradition de représentation des agents de malheurs qui a une série de parallèles déjà dans la Bible hébraïque, notamment en Ex 12 et 1 Ch 21, et qui est prolongée ailleurs dans la LXX, à savoir en Is 11 et dans les Psaumes de Salomon (Ps Sal 17).

Il faut enfin observer que le texte de la Sagesse montre un développement considérable par rapport à la LXX d'Habacuc. D'une part, le Logos y est représenté comme une hypostase de Yhwh, désormais étroitement liée à son trône. D'autre part, il est conçu comme une puissance à part entière, douée d'une force extraordinaire qui peut également être cause de mort et de destruction. Le Logos «démoniaque » est donc associé à Yhwh dans son rôle de gardien de l'ordre cosmique et exerce ce rôle dans les deux voies qu'il a à disposition : c'est-à-dire en établissant l'harmonie à travers l'acte de création, mais également en préservant l'ordre par la violence et la punition lorsque celui-ci risque d'être troublé.

$87 \quad$ Is $11,4 \mathrm{~b} \beta$.

88 Ps Sal 17,23-24, 35-36. 
Il est maintenant temps de revenir sur le deuxième passage, mentionné plus haut, qui atteste une lecture de deber comme dābār, à savoir le verset $6 \mathrm{du}$ Ps $90(=91 \mathrm{TM})$. Ce psaume constitue à bien des égards une pièce centrale $\mathrm{du}$ présent dossier. Il a, en effet, connu une très grande popularité dans les traditions magiques de l'Antiquité tardive, à la fois juives et chrétiennes. Il s'agit du texte biblique le plus fréquemment cité sur papyri, amulettes, bols incantatoires, phylactères, gemmes, bagues, et autres objets dits magiques. Il faut, en outre, souligner que les usages prophylactiques du psaume sont bien attestés autant pour le texte hébreu que pour sa version grecque; en outre, si l'incipit est la partie la plus souvent citée (verset 1 ou versets 1-2), d'autres versets ainsi que le psaume entier peuvent également être transcrits sur ce genre d'objets ${ }^{89}$.

Ne disposant pas d'informations concernant le contexte de composition ou d'usage du psaume, il n'est pas assuré que sa fonction originelle ait été spécifiquement incantatoire. Au contraire, la question de son Sitz im Leben demeure ouverte. Le texte hébreu constitue la pièce centrale d'un tryptique qui ouvre la quatrième collection des Psaumes $(90 ; 91 ; 92$ TM $=89 ; 90 ; 91$ LXX). Les trois textes sont construits autour du thème de la protection divine : à cet égard, le Ps 91 constitue une réponse à la requête d'aide formulée dans le psaume précédent tandis que le Ps 92 célèbre l'intervention de la divinité et la protection obtenue. La présence de plusieurs voix parlantes dans le texte ainsi que la référence à celui qui habite dans la demeure de la divinité et y passe la nuit au verset 1 pourraient appuyer l'hypothèse d'une origine dans la liturgie du temple, comme le suggèrent Hossfeld et Zenger ${ }^{90}$.

Néanmoins, plusieurs aspects thématiques et linguistiques du psaume ont pu favoriser sa réception en fonction apotropaïque. Tout d'abord, le texte oppose des puissances néfastes, porteuses de dangers et de maladies pour

89 Les témoignages papyrologiques et épigraphiques qui documentent l'usage du Ps 90 dans la version de la LXX en fonction apotropaïque ont été répertoriées pas Kraus 2005, 2007, 2018; voir également De Bruyn et Dijsktra 2011; Sanzo 2014, p. 106-120. Ils sont, pour la grande majorité, chrétiens même s'il faut tenir compte de la difficulté de trancher de façon rigide entre usages chrétiens, juifs, et «païens », au vu de la fluidité religieuse de premiers siècles de l'ère dans des contextes rituels de ce type : voir, à cet égard, les remarques de Boustan et Sanzo 2017. En revanche, je n'ai pas connaissance d'un répertoire des citations du texte hébreu. Sur les critères pour distinguer usages juifs et chrétiens du Psaume, voir Bohak 2008, p. 212-213. Sur les problèmes d'identification des éléments spécifiquement chrétiens sur les amulettes de l'Égypte gréco-romaine, voir De Bruyn et Dijsktra 2011, p. 168-171; Sanzo 2014, p. 10-14. Sur l'histoire de la réception du Ps 91 (= 9o LXX), voir Breed 2014 .

Hossfeld et Zenger 2005, p. 428. 
l'homme (versets 3-6), aux anges envoyés par Yhwh pour défendre le fidèle (versets 11-12). En outre, les dangers auxquels les humains sont confrontés sont représentés par des animaux farouches, étroitement associés aux forces chaotiques, tels que lions, serpents et dragons (verset 13, voir notamment la mention du tannîn), qui seront piétinés. L'auteur réadapte ici un sujet classique de l'iconographie proche-orientale qui célèbre le roi luttant contre les puissances du chaos ${ }^{91}$. Un thème ultérieur qui peut facilement se retrouver en contexte magique est celui de la connaissance du nom divin comme moyen d'activer la protection divine, au verset 14 : «Je le protégerai car il connaît mon nom ». Cette formule fait, d'ailleurs, écho à la concentration d'épithètes divines qui se trouve en ouverture (versets 1-2 : 'Elyôn, Shadday, Yhwh, 'Elohîm). Enfin, le psaume se termine par sept verbes ou expressions qui insistent sur la protection et les faveurs accordées par la divinité à son fidèle (« libérer », « protéger », « répondre », « délivrer», « honorer», « remplir les jours », « faire connaître le salut», versets $15^{-16)}$.

Il faut toutefois souligner que l'usage apotropaïque du psaume n'est pas un développement limité à l'Antiquité tardive. En effet, il est déjà attesté pendant la période du Second Temple, notamment à Qumrân. Deux versions de ce texte, datant du premier siècle de notre ère, ont été retrouvées parmi les manuscrits de la mer Morte. La première $\left(4 \mathrm{Q} 84=11 \mathrm{QPs}{ }^{\mathrm{b}}\right)$ est représentée par deux fragments qui reproduisent les versets $5^{-8}$ et $12-15$ : le texte est ici identique au texte massorétique ${ }^{92}$. La deuxième version a été recopiée dans le rouleau qu'on appelle des Psaumes apocryphes $\left(11 \mathrm{Q} 11={ }_{11} \mathrm{QPsAp}^{\mathrm{a}}\right)^{93}$ où elle fait suite à trois psaumes non attestés dans le texte massorétique. D’après une indication contenue en ${ }_{11} \mathrm{QPs}^{\mathrm{a}} 27$, aux lignes 9-10, ils avaient été composés par David pour être chantés «sur les possédés» $(\operatorname{lngn} \text { 'l pgw'ym })^{94}$. Les trois

91 Pour ce thème, voir l'étude classique de Keel 1993. Sur les connotations monstrueuses du tannîn, voir Angelini 2018 a, p. 26-33.

92 Skehan, Ulrich, et Flint dans Ulrich et al. 200o, DJD 16, p. 23-48. Le rouleau contient des fragments des Ps 91 à 118, dans un ordre différent de celui du TM. Le Ps 91 est suivi par le Ps 92 .

93 Editio princeps : Van der Ploeg 1965, 1971; je me réfère ici à l'édition de García Martínez, Tigchelaar et Van der Woude 1998 (DJD 23, p. 181-206). Ce rouleau a reçu une grande attention de la part des chercheurs, notamment à cause de la présence, à l'intérieur, du Ps 91. Parmi les études les plus importantes, voir Sanders 1997; Alexander 1999; Puech 2000 ; Lichtenberger 2003; Eshel 2003 a ; id. 2003 b ; Henze 2005 ; Pajunen 2008 ; Körting 2010 ; Evans 2011. L'origine sectaire du texte est acceptée par Alexander (1999, p. 345), mais contestée par d'autres chercheurs (notamment Eshel 2003 a ; id. 2003 b). Pour une discussion, voir Henze 2005, p. 88 sqq.

94 Voir également l'expression hpgwym dans ${ }_{11 Q P s} \mathrm{Ap}^{\mathrm{a}}$, fr. 4, col. v., l. 2. Une expression très similaire est utilisée à propos du Ps 91 dans le Talmud babylonien (b.Šebu.15b) : ושיר של 
premiers textes ont un langage exorcistique explicite : ils contiennent des formules qui deviendront typiques des incantations araméennes (notamment les expressions lḥ̌s, «incantation» et mšby, «j'adjure » $\left.{ }^{95}\right)$; ils mentionnent plusieurs démons (šédîm), le serpent-monstre marin (tannîn), Belial et d'autres puissances négatives; et l'un d'entre eux cite également le nom de Salomon. D'après Émile Puech et d'autres chercheurs, le rouleau semble avoir été organisé comme un rituel d'exorcisme ${ }^{96}$. Cette version du Ps 91 montre également un nombre assez élevé de variantes par rapport au texte massorétique, dont la plupart semblent relever d'un travail d'édition ${ }^{97}$. S'il est vrai qu'aucune de ces variantes n'accentue véritablement la dimension démonologique du psaume, sa position en fin de rouleau ainsi que la dernière formule qui fait référence à une récitation collective de ces textes (« et ils répondront: amen, amen ${ }^{98}$ ), semble indiquer que sa fonction était celle d'une prière apotropaïque ${ }^{99}$ à réciter pour clore une série d'exorcismes.

Un témoignage qui doit être mis en parallèle avec les fragments de Qumrân est celui du Nouveau Testament. Des passages de ce psaume dans sa version grecque sont cités ou référencés à plusieurs reprises dans les Évangiles, toujours dans des contextes où il est question de soumettre des démons: Luc $(10,17-19)$ et probablement Marc $(16,18)$ font référence à la capacité de fouler serpents venimeux et scorpions, en évoquant ainsi le verset 13. L'épisode des tentations infligées à Jésus par le diable dans le désert (Lc 4,10-11 et Mt 4,6) cite, en outre, littéralement les versets 11 et $12^{100}$. Un tel emploi a d'ailleurs très probablement influencé le développement du psaume comme texte magique dans les traditions chrétiennes antiques.

Il nous faut donc considérer la traduction grecque du Ps 91 (= 9o LXX) dans le contexte plus large de sa réception ancienne. La présence de plusieurs versions du texte à Qumrân soulève notamment la question de la Vorlage du traducteur grec. L'analyse des témoins disponibles semble suggérer que le traducteur lisait un texte assez proche du texte massorétique, au moins dans les six premiers versets qui nous intéressent particulièrement. Il faut néanmoins observer que

«le chant des possédés, et il y en a certains qui l’appellent 'chant des plaies'»).

95 Par exemple fr. 4 col. I l. 7 ; col. IV, l. 1. Voir Eshel 2003 a, p. 85-88; id. 2003 b, p. 403.

96 Puech 2000, p. 180-181; Lichtebenger 2003; Eshel 2003.

97 Pour une analyse détaillée des variantes, voir García Martínez, Tigchelaar et Van der Woude 1998 (DJD 23, p. 181-206); Pajunen 2008; Körting 2010.

$98 \quad$ 11Q11, fr. 4 col. vi, l. 14.

99 J'emprunte cette expression à Esther Eshel (2003 a), d'après laquelle le texte aurait été intentionnellement édité dans ce but.

100 Voir, à ce sujet, Henze 2005 et Evans 2011. 
le grec comporte une superscription attribuant le psaume à David ( $\alpha$ îvos $\dot{\omega} \delta \hat{\eta} \varsigma$ $\tau \hat{\omega} \Delta \alpha v i \delta)$. Celle-ci est absente du texte massorétique, mais pourrait avoir été présente déjà en $11 \mathrm{QPs}^{\mathrm{a} 101}$. Cette attribution est significative dans la mesure où, comme nous le verrons dans les chapitres suivants, David est un exorciste particulièrement doué dans les traditions bibliques. Une comparaison détaillée des passages en hébreu et en grec permet d'évaluer plus précisément le travail du traducteur :

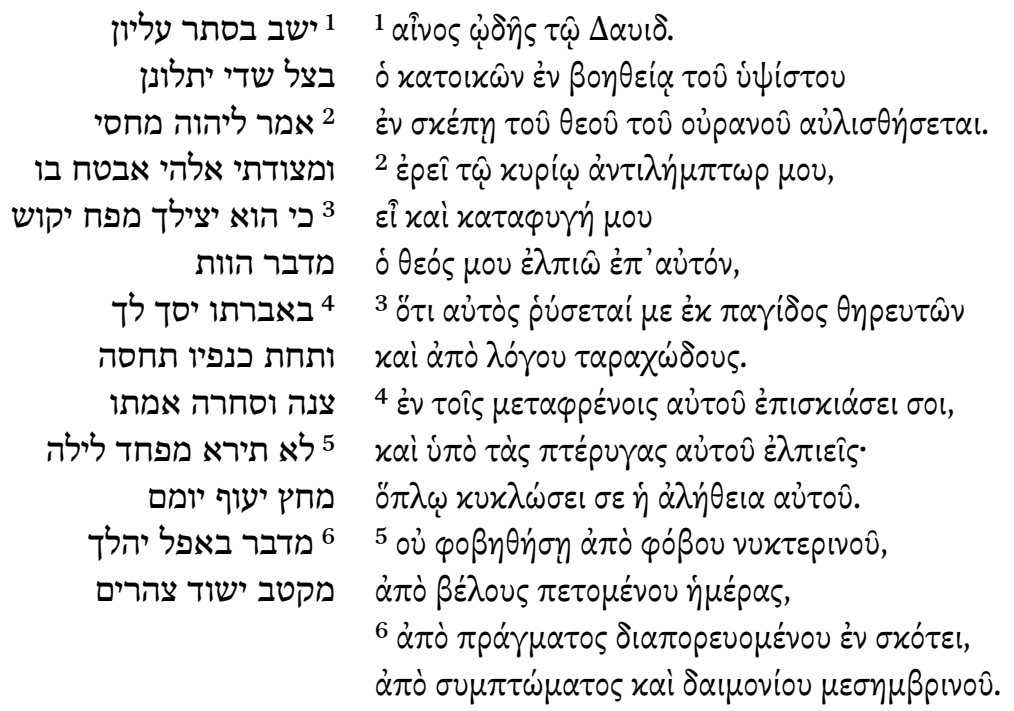

${ }^{1}$ Celui qui habite dans le (lieu) $\quad{ }^{1}$ Louange d'ode, de David.

secret du Très Haut,

à l'ombre de Shadday il loge,

${ }^{2}$ Qui dit ${ }^{102}$ à Yhwh : «mon

refuge,

ma forteresse, mon dieu, sur lui

Celui qui réside dans l'aide du Très Haut, logera sous la protection du dieu du ciel.

2 Il dira au Seigneur : «tu es mon

protecteur, mon refuge,

mon dieu, j'ai mon espoir en lui, je compte».

101 Fr. 4, col. vi, l. 2 : d'après les éditeurs, l'espace vide avant le premier mot du verset 1 pourrait être soit un vacat signalant la pause, soit un espace qui contenait un mot. Par analogie avec le psaume précédent qui mentionne David (col. v, l. 4), ils proposent de reconstruire ldwd (García Martínez, Tigchelaar et Van der Woude 1998, DJD 23, p. 204). Contrairement à la comparaison entre la version qumrânienne et celle massorétique du psaume, qui a reçu beaucoup d'attention de la part des chercheurs, une évaluation systématique des différences entre le texte grec et les versions hébraïques manque encore.

102 La vocalisation massorétique est à la première personne du singulier ('ōmar), ce qui implique un changement de sujet par rapport au verset précédent, alors que la LXX et la 
${ }^{3}$ C'est lui qui te délivre du filet du chasseur et de la peste ruineuse.

4 De ses ailes il te protège, et sous ses plumes tu te réfugies.

Sa fidélité est un bouclier et une armure.

5 Tu ne craindras ni la terreur de la nuit, ni la flèche qui vole au grand jour,

${ }^{6}$ ni Deber qui rôde dans

l'ombre,

ni Qețeb qui ravage à midi.
${ }^{3}$ car lui, il me libèrera du piège des chasseurs et d'un discours troublant ».

${ }^{4}$ Et il te fera ombre entre ses épaules et sous ses ailes tu trouveras l'espoir, par un bouclier sa vérité t'entourera.

5 Tu ne seras pas terrorisé par la terreur nocturne, par la flèche qui vole pendant le jour,

${ }^{6}$ par la chose qui marche dans l'ombre, par la chute et par le démon de midi.

Dans ces versets, le texte hébreu présente trois images centrées sur le thème de la protection de Yhwh contre différents dangers mortels qui sont ensuite prolongées dans le reste du psaume. La première image s'appuie sur la métaphore animalière de l'oiseau qui protège ses petits des pièges du chasseur; la deuxième adopte un langage militaire pour suggérer l'attaque des ennemis par la référence au bouclier ; la dernière évoque la panique $(p h d)$ générée par l'arrivée des plaies et maladies amenées par Deber et Qețeb. Ces trois représentations sont étroitement entrelacées : par exemple, la destruction amenée par la peste est annoncée au verset $3 \mathrm{~b}$ par l'expression middeber hawwwôt et, ensuite, reprise au verset 6 . En outre, l'image de la flèche volante au verset 5 s'inscrit à la fois dans la métaphore ornithologique et dans la métaphore militaire; elle sert également à introduire le thème du verset suivant qui porte sur l'arrivée des maladies conçues comme des «flèches » lancées contre l'homme : cette image rappelle de près celle de Deut 32 discutée plus haut. Deber et Qețeb sont ici sujets de verbes d'action et de mouvement, tels que $h l k$, « rôder », et $\check{s} d d$, «détruire », « dévaster», confirmant leur rôle de puissances porteuses de maladie et de mort (voir également l'usage attributif de hawwôt, « destruction », au verset 3). Le fait que Resheph ne soit pas nommé ne constitue pas un obstacle à la compréhension du passage en ce sens, comme cela a parfois

version qumrânienne lisent le verbe à la troisième personne, respectivement comme un futur ( $\left(\dot{p} p \varepsilon \hat{\imath}=y \bar{o}^{\prime} m a r\right)$, ou un participe $\left(h^{\prime} w m r\right)$. Matthias Henze (2005, p. 177-178) suggère une vocalisation originelle 'ōmèr. 
été soutenu ${ }^{103}$. Tout d'abord, il n'est pas rare que les agents de malheur soient nommés par couple plutôt que tous ensemble; deuxièmement, et comme je l'ai dit précédemment, à la lumière des parallèles avec Deut 32,22-24 on ne peut pas complètement exclure que «la flèche qui vole » mentionnée au verset 5 soit une référence à Resheph. En effet, ce qui différencie l'imaginaire du Ps 91 par rapport aux représentations de Deut 32 et d'Hab 3 ce n'est pas l'absence ou la présence de Resheph, mais le fait que les puissances évoquées dans le psaume ne sont plus décrites comme étant au service de Yhwh.

Bien que le traducteur n'ait pas traduit littéralement les noms de Deber et Qețeb, la traduction grecque préserve fidèlement les trois images du texte hébreu. Là, $d b r$ a été compris comme dābār dans le sens de "parole» au verset 3 b, et de «chose » $(\pi \rho \hat{\gamma} \gamma \mu \alpha)$ au verset 6 . Cette « chose » qui «marche dans

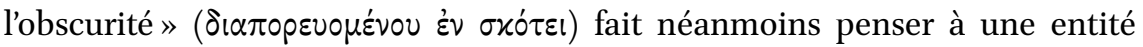
malfaisante, surtout par son association à une maladie : $\sigma \dot{u} \mu \pi \tau \omega \mu \alpha$ qui traduit qețeb et signifie généralement « accident», «malheur ». Mais, au sens strictement médical, $\sigma \dot{\nu} \mu \pi \tau \omega \mu \alpha$ peut aussi indiquer l'évanouissement et plus largement les symptômes d'un état altéré du corps. Cet usage, encore absent des traités hippocratiques, est par ailleurs bien préservé dans les textes médicaux hellénistiques ainsi que dans les papyri égyptiens du début de notre ère ${ }^{104}$.

La lecture de qețeb comme maladie est déjà attestée par la Lxx de

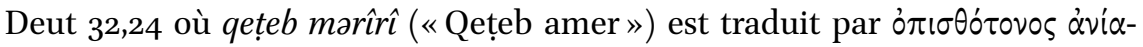
$\tau \circ \varsigma$, « convulsion incurable». Cette traduction semble faire référence à une maladie incurable caractérisée par la tension et la torsion de muscles car ỏ $\pi l-$

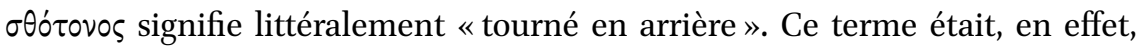
employé dans la littérature médicale ancienne pour indiquer les spasmes de l'épilepsie et surtout du tétanos, puisqu'il indique toute torsion des nerfs ${ }^{105}$. Ce choix pourrait donc dériver d'une lecture de la racine $q t \underline{b}$ dans son sens premier de «tailler », « piquer », en faisant référence à la manière dont on attrape le tétanos. Cette lecture semble déjà attestée par la LXX d'Os 13,14, où qețeb est nommé parmi les calamités associées au Sheol et à la mort et où il est traduit en grec par $\chi \dot{\varepsilon} v \tau$ pov, « aiguillon » :

103 Frey-Anthes 2007, p. 97-98, suivie par Körting 2010, p. 573-574.

104 Sor. 1, 48; P. Wash. Univ 2 67, l. 12-13; P. Tebt 2.272, l. 11 (sur ce dernier voir Marganne 1981). En revanche dans le Corpus hippocraticum le terme apparaît seulement deux fois dans le sens classique d'« accident» (Hp., Decent. 6, $2 ; E p .16,48)$.

105 Voir, par exemple, la description de ces maladies donnée par Platon (Ti. 84e); ou Dscr. 3, 8o, 5. Sur ce sujet, voir Dogniez 2003. 


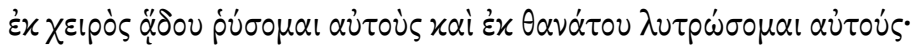

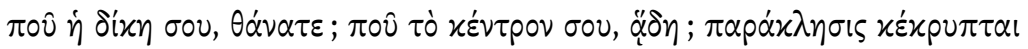
$\dot{\alpha} \pi \dot{\partial} \partial \varphi \theta \alpha \lambda \mu \hat{\omega} v \mu \circ v$.

Je le libérerai de la main d'Hadès et je les rachèterai de la mort.

Où est ton jugement, mort ? Où est ton aiguillon, Hadès? Tout confort se cache de mes yeux.

On pourrait donc avancer l'hypothèse que ces lectures relèvent d'une oblitération des croyances concernant l'identité spécifique de puissances telles que Qețeb et Deber; puissances dont l'identité est par ailleurs assez opaque dans la Bible hébraïque. Toutefois, leur association à des maladies est conservée dans la plupart des occurrences. Dans le cas particulier du Ps 9o, le traducteur a bien saisi le côté malfaisant de ces puissances pouvant être responsables de malheurs et il met en évidence cet aspect. De plus, dans ce contexte, on voit apparaître pour la première fois le démon de midi. Comme je l'ai déjà souli-

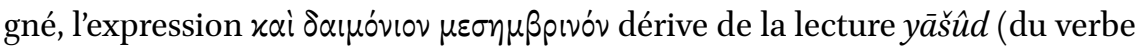
šdd, « détruire », « dévaster ») comme wašyd, « et le démon », šêd étant dans d'autres passages de la Bible hébraïque le nom générique pour indiquer les

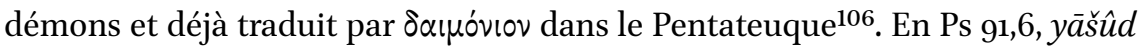
est certainement à considérer comme la leçon originelle pour deux raisons : d'abord cette forme verbale permet de compléter le parallélisme des membres des versets 5 et $6^{107}$. En outre, en araméen et en hébreu tardif l'orthographe régulière pour démon est notamment šyd, ce qui a probablement influencé la lecture du traducteur ici.

L'idée d'un démon qui apparaît à midi n'a pas de véritables antécédents dans les traditions levantines ni proche-orientales, mais elle est compréhensible à la lumière du contexte grec et plus spécifiquement hellénistique. Traditionnellement, le midi était une heure redoutable car ouverte aux passages de fantômes et autres créatures que l'on peut volontiers qualifier de «démoniaques», comme par exemple Empousa ${ }^{108}$. Pour les anciens, à cette heure, Pan et les Nymphes, censés être capables de conduire à des états de possession et de provoquer des crises épileptiques, étaient considérés comme particulièrement dangereux. Une Idylle du poète hellénistique Théocrite nous

106 Voir supra, § 3.1.

107 Comme le relève justement Schaper 2001, p. 178.

108 Schol. in Ar. Ranas 293. Pour Empousa, voir p. 55-57. D’après Servius, (in Georg. 4, 400) midi est l'heure de la manifestation des numina. Dans ses Antiquités Romaines, Denys d'Halicarnasse raconte que Servius Tullius s'endormit à midi et un feu lui apparut sur la tête comme signe de sa royauté $(4,2,4)$. 
en donne témoignage. Ici, un pasteur met en garde son compagnon contre les périls du midi ${ }^{109}$ :

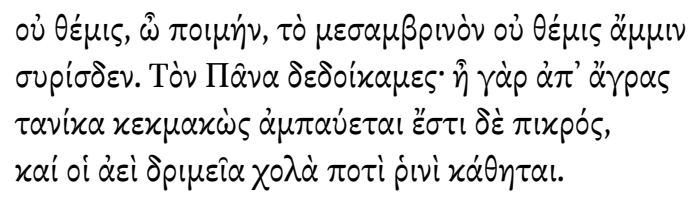

Il nous est interdit, ô berger, interdit, à l'heure méridienne, de jouer de la syrinx. Nous avons peur de Pan. C'est le moment où, après la chasse, lassé il se repose. Son humeur est colère; et toujours l'âcre bile est prête à lui monter au nez.

C'est donc dans cette tradition que puise le traducteur du Psaume. En outre, et comme observé précédemment, parmi les textes qui décrivent l'action des puissances de malheur le Ps 91 est le seul endroit où ces dernières sont présentées comme capables d'affliger l'individu dans sa propre singularité par le biais de maladies (qețeb/deber en hébreu) ou d'états altérés ( $\sigma \dot{\mu} \mu \pi \tau \omega \mu \alpha$ en grec), et ce, sans motivation évidente comme ce pouvait être le cas en Deut 32. Ainsi, elles semblent ici douées d'une agentivité qui leur est propre. Comme nous l'avons constaté, cette agentivité opère déjà dans le texte hébreu qui semble mettre en place une vision dualiste. En effet, à la différence des autres passages bibliques examinés, Deber et Qețeb n'agissent pas sous injonction divine directe, mais sont opposées à la divinité. De plus, leur action contraste avec celle des anges du verset 11 qui, eux, sont envoyés directement par Yhwh pour protéger le fidèle, en le soulevant littéralement dans leurs mains. Le traducteur grec semble donc s'inscrire à sa manière dans la ligne d'interprétation « exorcistique » du Ps 91, qui, comme nous l'avons vu, traverse toute l'Antiquité et se trouve déjà, en large mesure, préparée par le texte hébreu ${ }^{110}$.

Toutefois, il importe de souligner que, pour ce faire, le traducteur n'a pas juste emprunté les catégories grecques, mais il a innové en utilisant à la fois l'arrière-plan hébreu et le contexte grec. D'un côté, en contraste avec le texte hébreu, le traducteur introduit l'idée d'une puissance capable d'attaquer l'homme dans un moment spécifique de la journée. L'expression hébraïque

109 Theoc., Idylle 1 (Thyrsis), 15-18 (trad. Legrand, Paris, Les Belles Lettres, 1925).

110 Outre à Qumrân et dans le Nouveau Testament, cette interprétation est également attestée dans les Targums, dans la Peshitta et dans plusieurs traditions rabbiniques : b.Pesah. 111 ; ; b.Šebu. 15b ; y.Erub. 10,11 (26c) ; y.Šabb. 6,2 (8b) ; Midrash Tehillim 91, 5. Voir Henze 2005 ; Breed 2014. 
șohŏrāyim (verset 6, litt. «en plein midi») n'indique pas l'heure de l'attaque démoniaque : elle exprime plutôt, en alternance avec la référence à la nuit ( $b \bar{a}$ ôpel), l'idée d'une terreur constante dans le temps qui n'abandonne jamais l'homme. Cette alternance entre lumière et obscurité, nuit et jour, revient d'ailleurs dans les deux autres psaumes de la trilogie ${ }^{111}$. Ainsi faisant, le traducteur rejoint ou, pour mieux dire, anticipe une interprétation juive qui se développe de manière parallèle dans les traditions rabbiniques sur Qețeb, lesquelles opèrent la distinction entre un démon qui attaque le matin (le qețeb marîrî de Deut 32) et un démon ravageur de l'après-midi (qeteb yā̌šûd șohŏrāyim du Ps 91 $)^{112}$. D'un autre côté, il faut préciser que la notion de «démon de midi » était inconnue du monde grec. Non seulement l'expression apparaît pour la première fois dans la LXX, mais, de plus, une telle catégorie religieuse, qui, après avoir été reprise par Jérôme, aura une grande fortune chez les Pères ${ }^{113}$, n'a pas de véritable antécédent dans la religion de la Grèce ancienne. De manière générale dans les traditions plus antiques, tant au Proche Orient antique qu'en Grèce, le moment des attaques démoniaques se situe davantage pendant la nuit ${ }^{114}$.

Essayons, à présent, de résumer brièvement les étapes de ce parcours. En contraste avec le texte hébreu d'origine, les représentations d'entités démoniaques subissent une transformation partielle dans la LXx. Dans les contextes poétiques développés par Hab 3 et le Ps 90 (= $91 \mathrm{TM})$, le scénario de départ a été remplacé par un second scénario, nouveau. Celui-ci a gardé un certain souvenir des puissances auxiliaires de Yhwh ainsi que de leurs fonctions, tout en renforçant et spécifiant leurs caractères, et cela en dépit du fait de ne pas avoir reconnu l'identité spécifique à chacune de ces puissances, ni d'avoir conservé

111 Ps 90,4-6; 92,3.

112 b.Pesah 111 b; MidrashTehillim 91. De manière plus générale, les sources rabbiniques tendent à donner plus de précisions sur les circonstances temporelles et spatiales des attaques démoniaques ainsi que sur l'apparence physique des démons.

113 Chez les Pères de l'église ce type de démon serait notamment responsable de l'acedia qui saisit les moines. Il faut toutefois remarquer que le démon de midi ne semble pas avoir attiré l'attention des Pères avant le quatrième siècle, lorsque Jérôme et Augustin commencent à en discuter. Il se peut que l'association entre démon de midi et acedia remonte à Origène (Fr. in Ps. 5, 6), mais l'authenticité de ce texte est disputée. Voir à ce propos Arbesmann 1958; récemment Scott-Macnab 2018.

114 Comme l'a bien montré Philippe Borgeaud (2000), la prétendue continuité entre les démons anciens et les démons du midi connus par la tradition occidentale est surtout le produit de la plume de l'écrivain et sociologue Roger Caillois (1937) qui a projeté des croyances du Moyen Age slave sur l'Antiquité, sans qu'elles ne soient réellement soutenues par les sources antiques. 
un souvenir de l'arrière-plan levantin du dieu Resheph. Le Logos divin d'Hab 3 est, par exemple, une puissance à la fois céleste et destructrice qui exécute impitoyablement les ordres divins. La fonction de cette puissance est encore développée dans d'autres écrits du judaïsme hellénistique, représentées entre autres par le livre de la Sagesse qui se confrontent de manière directe à d'autres courantes philosophiques de lépoque, et qui visent à inscrire leurs propres discours au sein des débats intellectuels contemporains. Le rôle du mašhhit, comparable à celui du Logos en Habacuc et en Sagesse, est également bien préservé et compris par la tradition juive de langue grecque dans son ensemble. Le seul endroit où les assistants de Yhwh ont complètement disparu dans le texte grec est la LXX de Deut 32,24 où la divinité apparaît comme la seule responsable des châtiments, fléaux et maladies. Laction néfaste des auxiliaires de Yhwh a ici laissé la place à une représentation du châtiment divin qui est à la fois plus rationnelle et plus sanglante, où les cadavres des hommes tués par la famine seront consommés par des rapaces. Mais un tel glissement semble dû à une difficulté inhérente à la compréhension du texte de départ plutôt qu'à une correction théologique du traducteur. En effet, n'étant plus en mesure de reconstruire l'identité de Qețeb et Resheph, le traducteur se serait appuyé sur la lecture étymologique de la racine $q t \underline{b}$ ainsi que sur l'exégèse hébraïque du mot ršp : deux stratégies qui sont, me paraît-il, courantes lorsque les traducteurs se heurtent à des difficultés de vocabulaire, et conformes au style adopté par le traducteur du Deutéronome dans son ensemble. Ce dernier point me permet, entre autres, d'avancer une remarque plus générale concernant la nature de la LXX du Deutéronome, qui encore dans les débats récents est souvent étiquetée, peut-être un peu trop rapidement, comme «littérale » ou «peu soignée ». Les exemples analysés ici montrent en revanche que le souci du traducteur était moins celui de garder un littéralisme extrême que celui de produire un texte qui pouvait «fonctionner » en grec, tant du point de vue de la langue et du registre littéraire - qui en Deut 32 est un registre poétique - que du point de vue des concepts employés. Cette remarque confirme alors que la dichotomie entre traduction littérale et libre, dont j'ai déjà discuté dans l'introduction, ne constitue pas la meilleure démarche pour comprendre et décrire le travail des traducteurs grecs. En revanche, il faut s'orienter vers d'autres critères qui tiennent en compte non seulement du rapport de la traduction à son texte hébreu, mais également de ses potentialités de fonctionnement à la lumière du contexte linguistique et culturel grec.

En outre, en ce qui concerne les représentations du démoniaque, sur la base des résultats de notre analyse, nous pouvons donc conclure que l'idée, souvent proposée, selon laquelle la LXX aurait systématiquement cherché à 
démythologiser les scénarios de son texte source n'est pas tout à fait convaincante. Il apparaît, alors, nécessaire de s'orienter vers d'autres modèles pour interpréter l'ensemble de ces données. Le Ps 9o (= 91 TM) s'avère, en ce sens, particulièrement utile. Ici, l'association directe établie entre créatures nocturnes qui volent dans l'ombre, maladies et dangers du midi s'inscrit pleinement dans le contexte hellénistique où la notion de démon est en train de se construire. Le traducteur y apporte alors une contribution considérable, sans que cela soit dû au seul fait qu'il enrichisse le paysage religieux de l'époque du démon de midi. Par le biais de ce texte, il inscrit l'action des puissances démoniaques au sein d'une vision du monde essentiellement dualiste qui a peu d'antécédents dans la Bible hébraïque ${ }^{115}$ et qui est largement préparée, comme nous l'avons vu, par le texte hébreu du Psaume. Dans ce cadre, et contrairement à ce qui arrive en Deut 32,22-24, les démons assaillent l'homme de manière soudaine, sans motivation évidente et leur attaque est neutralisée par l'action des anges.

Il est néanmoins indéniable que le statut exceptionnel du Psaume ait exercé une influence sur la manière dont le rôle des démons y est représenté. Autrement dit, ce texte seul ne peut pas nécessairement être considéré comme donnant la mesure moyenne de l'engagement des traducteurs avec un savoir sur les démons diffusé à l'époque hellénistique. Pour avoir une appréciation globale de l'apport des traducteurs à la construction d'une notion partagée du démoniaque, il faut à présent examiner les autres contextes dans lesquels cette notion est opérationnelle.

115 Voir, à ce sujet, Keel 2003. 